\author{
MITSUBISHI ELECTRIC RESEARCH LABORATORIES
}

http://www.merl.com

\title{
Characterization of Flow-Magnetic Field Interactions in Magneto-Hydrodynamic Turbulence
}

\author{
Richard, J.C.; Kumar, G.; Kalmar-Nagy, T.; Girimaji, S.S.
}

TR2013-013 January 2013

\begin{abstract}
We examine the complex nonlinear flow-magnetic field dynamics in magnetohydrodynamic (MHD) turbulence. Using direct numerical simulations (DNS), we investigate the dynamical interactions subject to the influence of a uniform applied background magnetic field. The initial magnetic and kinetic Reynolds numbers (based on Taylor microscale) are 45 and there are no initial magnetic field fluctuations. The sum total of turbulent magnetic and kinetic energies decays monotonically. With time, the turbulent magnetic fluctuations grow by extracting energy from velocity fluctuations. Expectedly, the distribution of energy between kinetic and magnetic fluctuations exhibits large periodic oscillations from the equipartition state due to Alfven waves. We perform a detailed analysis of the flow-magnetic field coupling and posit a simple model for the energy interchange. Such dynamical analysis can provide the insight required for turbulence control and closure modeling strategies.
\end{abstract}

Journal of Computational and Nonlinear Dynamics

\footnotetext{
This work may not be copied or reproduced in whole or in part for any commercial purpose. Permission to copy in whole or in part without payment of fee is granted for nonprofit educational and research purposes provided that all such whole or partial copies include the following: a notice that such copying is by permission of Mitsubishi Electric Research Laboratories, Inc.; an acknowledgment of the authors and individual contributions to the work; and all applicable portions of the copyright notice. Copying, reproduction, or republishing for any other purpose shall require a license with payment of fee to Mitsubishi Electric Research Laboratories, Inc. All rights reserved.
} 

Jacques C. Richard ${ }^{1}$

Mem. ASME

Senior Lecturer

e-mail: richard@tamu.edu

\title{
Characterization of Flow-
}

Gaurav Kumar

Graduate Student

e-mail: gauravkr@neo.tamu.edu

Department of Aerospace Engineering,

Texas A\&M University,

College Station, TX 77843-3141

\author{
Tamás Kalmár-Nagy \\ Mem. ASME \\ Principal Member Research Staff \\ Mitsubishi Electric Research Laboratory, \\ Cambridge, MA 02139 \\ e-mail: jcnd@kalmarnagy.com
}

Sharath S. Girimaji
Mem. ASME
Professor
Department of Aerospace Engineering,
Texas A\&M University,
College Station, TX 77843-3141
e-mail: girimaji@aero.tamu.edu
Magnetic Field Interactions in Magneto-Hydrodynamic Turbulence

We examine the complex nonlinear flow-magnetic field dynamics in magnetohydrodynamic (MHD) turbulence. Using direct numerical simulations (DNS), we investigate the dynamical interactions subject to the influence of a uniform applied background magnetic field. The initial magnetic and kinetic Reynolds numbers (based on Taylor microscale) are 45 and there are no initial magnetic field fluctuations. The sum total of turbulent magnetic and kinetic energies decays monotonically. With time, the turbulent magnetic fluctuations grow by extracting energy from velocity fluctuations. Expectedly, the distribution of energy between kinetic and magnetic fluctuations exhibits large periodic oscillations from the equipartition state due to Alfvén waves. We perform a detailed analysis of the flow-magnetic field coupling and posit a simple model for the energy interchange. Such dynamical analysis can provide the insight required for turbulence control and closure modeling strategies. [DOI: 10.1115/1.4023323]

\section{Introduction}

The nonlinear physical phenomenon of turbulent fluid flow is of great importance in nature and industrial applications. The multiscale, multiphysics interactions that characterize turbulence render it inherently complex. The nonlinear interactions among the various scales of turbulent motion lead to further proliferation of scales-a process known as energy cascade. The nonlinearity in the governing equations also leads to chaotic behavior and intermittency. In magneto-hydrodynamic (MHD) turbulence, the flow field evolution is significantly influenced by the magnetic field through the so-called Lorentz force. Like many nonlinear systems, MHD turbulence displays a tendency toward energy equipartition [1-5]. The objective of this study is to examine the nonlinear coupling between velocity and magnetic field fluctuations for the purpose of developing a fundamental understanding of MHD turbulence. We particularly seek to pursue a dynamical system approach to this study for developing simple models of interaction and ultimately utilize this insight to constructing turbulence control strategies.

Magneto-hydrodynamics [6] is the study of electrically conducting fluids such as plasmas, liquid metals, liquid superconductors, and ionic solutions. An imposed external magnetic field can induce a current in a moving conducting fluid (as per the laws of Faraday and Lenz), which, in turn, creates body forces in the fluid. The body force can significantly modify the fluid motion, rendering it very different from pure hydrodynamic flow. Furthermore, the flow of a conducting fluid generates a secondary magnetic field that changes the total magnetic field lines from that due to the imposed background field. Thus, in MHD turbulence, there exists a strong coupling between the Navier-Stokes equations that govern hydrodynamic motion and Maxwell's equations of electro-

\footnotetext{
Corresponding author.

Contributed by the Design Engineering Division of ASME for publication in the Journal of Computational and Nonlinear Dynamics. Manuscript received January 4, 2012; final manuscript received December 12, 2012; published online January 25, 2013. Assoc. Editor: D. Dane Quinn.
}

magnetism. The coupling can lead to many interesting phenomena not witnessed in hydrodynamic flows.

Much like hydrodynamic flow, MHD flows become turbulent at high Reynolds numbers [7,8]. Magnetohydrodynamic turbulence plays a major role in many flows in nature and engineering: e.g., electric propulsion, flow control, and materials. Electric propulsion (EP) concepts utilize electric and magnetic fields to direct the flow of plasma to generate thrust. Such propulsion concepts include ion thrusters [9], Hall thrusters [10], and VASIMR (Variable Specific Impulse Magnetoplasma Rocket) [11]. Turbulence influences plasma jet production and propagation in EP [11,12]. The flow-magnetic field coupling determines how well MHD generators and accelerators perform in different conditions [13].

A comprehensive review of MHD turbulence can be found in the referenced articles $[3,4,6,8,14-29]$. Depending on the magnetic field configuration, the Lorentz forces can alter the coherent structures in turbulent flow by generating flows with or against the hydrodynamic tendency [14]. It can also redistribute energy and momentum in the system between the kinetic and magnetic energy modes. In the absence of mean velocity gradients, MHD turbulence decays with time (since there is then no turbulence production). This energy decay exhibits many interesting features depending upon the parameter regime of the flow.

The focus of this paper is on the dynamics of flow-magnetic field coupling, specifically magnetic-kinetic energy exchange brought about by the Lorentz force. In particular, we will investigate the coupling in the presence of a uniform magnetic field. Toward this end, we perform direct numerical simulations (DNS) of decaying MHD turbulence with an imposed uniform external field. We perform an analysis of the coupling and develop a simple dynamical model that replicates observed behavior. Furthermore, we try to develop a physical understanding underlying the observed dynamics.

In Sec. 2, we present the governing MHD equations used in this study and discuss the various turbulence decay computations performed. Section 3 contains the detailed results from the simulations. In Sec. 3.2, we present the analysis and provide a simple 
phenomenological model. The physics underlying the dynamics is investigated in Sec. 4. The conclusions are given in Sec. 5.

The physical investigation and dynamical analysis presented in this paper is also important in the context of MHD turbulence closure model development. Turbulence modeling of MHD flows would entail closures for kinetic/magnetic dissipation and Lorentz work terms. The standard dissipation closure models are contingent on kinetic energy being an inertial scale invariant. However, in MHD turbulence, it is only their total energy that is an invariant in the absence of inviscid effects. Here we develop a simple dynamic model for the turbulent kinetic and magnetic energies. Such a model will serve as the foundation of future closure models for kinetic/magnetic dissipation and Lorentz work.

The closure coefficients of the dynamic model developed in this work are specific to the parameters and initial conditions used in the simulations. Clearly, the overall procedure is of most general applicability and the closure coefficients can be rendered functions of the dimensionless parameters as more DNS and experimental data become available.

\section{Governing Equations and Simulations}

The equations used in the current investigation are those that govern incompressible MHD flows [6]:

$$
\begin{gathered}
\nabla \cdot \mathbf{v}=0 \\
\rho \frac{\partial \mathbf{v}}{\partial t}+\rho(\mathbf{v} \cdot \nabla) \mathbf{v}=-\nabla p-\frac{\nabla(\mathbf{B} \cdot \mathbf{B})}{2 \mu_{0}}+\frac{\mathbf{B} \cdot \nabla \mathbf{B}}{\mu_{0}}+\mu \nabla^{2} \mathbf{v} \\
\frac{\partial \mathbf{B}}{\partial t}+(\mathbf{v} \cdot \nabla) \mathbf{B}=(\mathbf{B} \cdot \nabla) \mathbf{v}+\eta \nabla^{2} \mathbf{B} \\
\nabla \cdot \mathbf{B}=0
\end{gathered}
$$

where $\mathbf{B}$ is the magnetic induction field, $\mathbf{v}$ is the velocity field, $\rho$ is density, $p$ is pressure, $\mu$ is dynamic viscosity, $\mu_{0}$ is the magnetic permeability of free space, and $\eta$ is magnetic diffusivity related to the conductivity: $\sigma=1 / \mu_{0} \eta$.

The key quantities of interest in this study are the turbulent kinetic energy, magnetic energy, and Lorentz work [3,15,29]:

$$
\begin{gathered}
E_{K}=\int \frac{v^{\prime 2}}{2} d V \\
E_{M}=\mu_{0} \int \frac{B^{\prime 2}}{2} d V \\
E_{L}=\int \mathbf{v}^{\prime} \cdot\left(\mathbf{j}^{\prime} \times \mathbf{B}^{\prime}\right) d V
\end{gathered}
$$

The current density $\mathbf{j}$ is given by

$$
\mathbf{j}=\nabla \times \mathbf{B} / \mu_{0}
$$

Primed quantities are fluctuating components and over-bars are averages, e.g., $\mathbf{B}=\overline{\mathbf{B}}+\mathbf{B}^{\prime}, B^{\prime}$ is the magnitude of $\mathbf{B}^{\prime}$.

Evolution of hydrodynamic turbulence and kinetic-magnetic field interactions influence the decay of kinetic and magnetic energies. The total energy, without any source, decays at a rate dependent on two parameters: the Taylor Reynolds number, $\operatorname{Re}_{\lambda}=v_{\text {rms }}^{\prime} \lambda / \nu,(\nu=\mu / \rho, \lambda$ is the Taylor microscale [6]), and magnetic Reynolds number, $\operatorname{Re}_{m}=\operatorname{Re}_{\lambda} \nu / \eta$. We can quantify the interaction of the two fields in terms of the so-called "interaction parameter" also known as the Stuart number:

$$
N=\sigma \bar{B}^{2} \lambda / \rho v_{\mathrm{rms}}^{\prime}
$$

The kinetic and magnetic energy evolve as:

$$
\begin{gathered}
\frac{\partial}{\partial t}\left(\rho \frac{v^{2}}{2}\right)=\mathbf{v} \cdot \nabla\left(\rho \frac{v^{2}}{2}-p\right)+\mathbf{v} \cdot(\mathbf{j} \times \mathbf{B})+\mathbf{v} \cdot \mu \nabla^{2} \mathbf{v} \\
\frac{\partial}{\partial t}\left(\frac{B^{2}}{2 \mu_{0}}\right)=-\frac{1}{\mu_{0}} \nabla \cdot(\mathbf{E} \times \mathbf{B})-\mathbf{v} \cdot(\mathbf{j} \times \mathbf{B})-\mu_{0} \eta j^{2}
\end{gathered}
$$

where the electric field, $\mathbf{E}$, is related to the current density as

$$
\mathbf{E}=\mathbf{j} / \sigma-\mathbf{v} \times \mathbf{B}
$$

The Lorentz work term, $\mathbf{v} \cdot(\mathbf{j} \times \mathbf{B})$, appears with opposite sign in the kinetic and magnetic energy equations (Eqs. (10) and (11)). This is the key mechanism for energy exchange between velocity and magnetic fields. The energy exchange is also observed as the action of Alfvén waves [22,30].

2.1 Computational Approach: The Lattice Boltzmann Method. The computational algorithm employed in this study is the lattice Boltzmann method (LBM) [31-45]. The lattice Boltzmann equation is a discretized form of the Boltzmann equation of statistical thermodynamics. Equations (1) and (2) may be derived from Boltzmann's equation using the Chapman-Enskog expansion [34,35]. A vector analog yields Eq. (3) and satisfies Eq. (4) [46]. Macroscopic properties (e.g., $\rho, \rho \mathbf{v}, \mathbf{B}$ ) are computed as moment integrals of the velocity distribution functions obtained from solving these equations. These linearized Boltzmann-type equations are integrated to second order along each equation's discrete characteristic velocity sets. First, the velocity space is discretized into a set of small number and highly symmetric points so that a sufficient number of the members of the associated velocity distribution function are preserved exactly by the numerical quadratures. Second, the discrete velocity set co-locates points in a lattice in space so that the numerical integration consists of two simple steps: collision (local) and advection (to nearest neighbor lattices) [34,35]. Polynomial representation of the equilibrium distributions and corresponding times to relax to equilibrium (that also yield the diffusive coefficients) are sufficient to accurately resolve macroscopic properties of interest [46].

In Refs. [36] and [41], DNS of decaying isotropic turbulence is performed and all of the critical physics including power-law decay are clearly demonstrated. Effects of temperature fluctuations [36] and system rotation [43] are also accurately replicated. In Ref. [44], LBM-DNS is performed for time-varying homogeneous shear flow. In [45], LBM-LES (large-eddy simulation) is performed for rectangular and square jets. In summary, LBM is well established as an excellent computational tool for DNS and LES of turbulence.

Successful MHD-LBM computations require three key elements: (i) a strategy to account for the effect of the magnetic field on the velocity field; (ii) a means of solving the magnetic induction equation; and (iii) a velocity field solver. We use the fully-validated MHD-LBM computer code of Riley et al. [12,47] based on the discretized magnetic field scheme proposed by Dellar [46]. Riley et al. [47] establish the accuracy and robustness of this method for MHD turbulence simulations.

For example, the MHD-LBM calculations of the Hartmann flow is performed and compared against analytical data resulting in precise agreement [48]. Rectangular plasma jet vorticity dynamics have been resolved using LBM $[12,47]$. Turbulent flow characteristics have been quantified using LBM [36,41-45,49] and compares very well with DNS based on the spectral method [50-52]. More importantly, MHD turbulence calculations in Ref. [25] show that the vorticity budget and turbulence smallscale structure (orientation between vorticity and strain-rate eigendirections) are well-captured. Important magnetic field invariants such as helicity and cross-helicity are also investigated. At this 
stage of development the MHD-LBM code is well-proven for further investigations.

2.2 DNS Parameters. We conduct studies of uniform mean magnetic fields of strengths of $0.001 \mathrm{~T}$ and $0.0025 \mathrm{~T}$, for which the interaction parameter are $N=0.05$ and 0.3 , respectively. The initial strength and the alignment of the magnetic field relative to velocity fluctuations strongly influence the evolution of MHD turbulence. In the present simulations, the magnetic field is initially uniform with no fluctuations. Thus the velocity field interactions with the uniform background field solely initiate magnetic fluctuations. Subsequent evolution of magnetic fluctuations depends on the nonlinear interplay between velocity and magnetic fluctuations.

In all simulations, the velocity field is initially random, isotropic and incompressible, specified as discussed in Kerimo and Girimaji [53]. The initial isotropic velocity field has an energy spectrum of the form [5]

$$
E_{K}(\kappa, 0) \propto \kappa^{4} e^{-2\left(\kappa / \kappa_{0}\right)^{2}}
$$

Initially, only wave numbers $\kappa=2,3,4$ are energized. The initial Taylor Reynolds and magnetic Reynolds numbers are both 45. The magnetic Prandtl number is unity. It is clearly demonstrated in Ref. [25] that all of the important nonlinear aspects of turbulence are already in evidence at this relatively low Reynolds number of 45 . For example, the correct power-law exponent is seen in LBM-DNS. Even more importantly, it is seen that vorticity aligns principally with the intermediate strain-rate eigendirections, which is an important signature of small-scale effects. This is the range of Reynolds and Prandtl numbers investigated in Refs. [19], [20], and [23]. Thus, the dimensionless parameters used in the current study are well in keeping with relevant physics and other works in literature. The value of resolution indicator $\kappa_{\max } \eta_{\kappa}$ is about 2 during the peak of cascade, where $\eta_{\kappa}$ is the Kolmogorov microscale. This indicates that the smallest scales are well resolved. The computational domain is a $128^{3}$ uniform mesh with periodic boundaries.

\section{Analysis of Simulation Results}

One of the most important features of MHD turbulence is the coupling dynamics between flow and magnetic fluctuations [25] leading to energy interchange. The energy exchange is the focus of this work. Previous studies [25] have been performed to understand the dynamics of this exchange in decaying homogeneous MHD flows with and without imposed background magnetic fields. The energy exchange in the absence of an imposed background magnetic field is straightforward: there is a monotonic evolution toward kinetic-magnetic energy equipartition. However, the presence of a background magnetic field complicates the flowmagnetic field coupling. Some of the important findings for the case of turbulence decay under the influence of a uniform background magnetic field with no initial magnetic fluctuations are summarized in our previous study [25]:

(1) Initially, the Lorentz force rapidly transfers energy from velocity to magnetic fluctuations. Indeed, the magnetic energy overshoots kinetic energy. Subsequently, the Lorentz force tries to impose equipartition. The evolution toward equipartition is oscillatory with intermittent large fluctuations. Alboussiérre et al. [1] have also observed these oscillations in their numerical and experimental work.

(2) Over time, the amplitude and frequency of the oscillations in kinetic and magnetic energy decrease, partly due to kinetic energy decay, and partly due to the initially isotropic fluctuating velocity field adjusting to the directionality of the mean magnetic field (see Figs. 1 and 2).

(3) Comparing MHD turbulent flows with different uniform mean magnetic field strengths, the so-called interaction

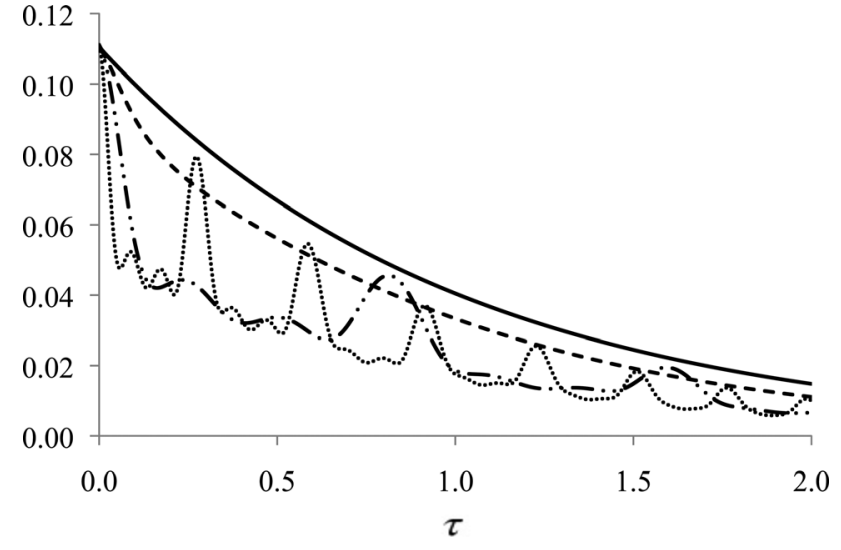

Fig. 1 Kinetic and Magnetic Energy Decay with eddy turnover

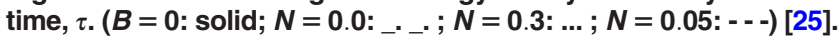

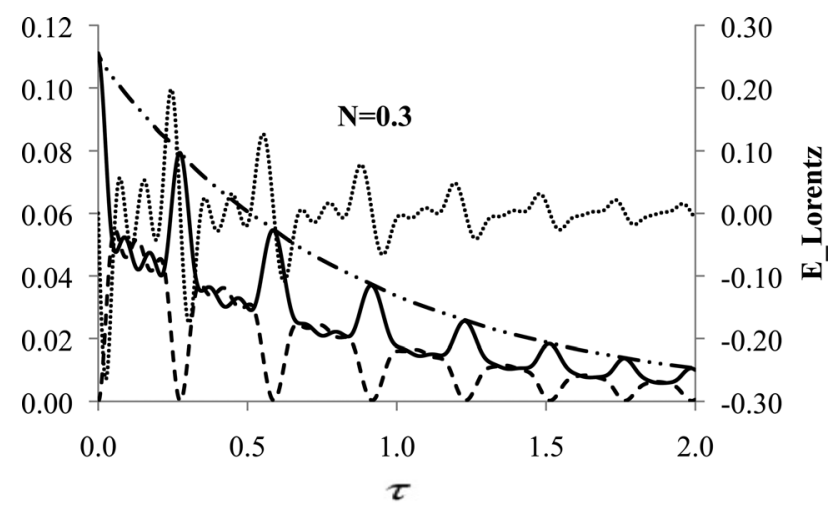

(a)

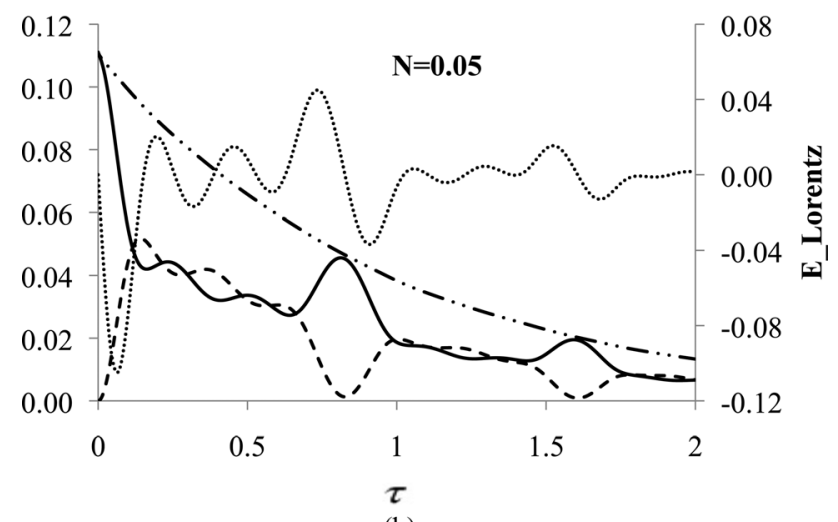

(b)

Fig. 2 Evolution of $E_{M}, E_{K}, E_{t}$ and Lorentz work with $\tau$. (a) $N=0: 3$; (b) $N=0: 05$ (solid: Kinetic Energy; _._. : Total Energy; ... : Lorentz work, - - - : Magnetic Energy) [25].

parameter was demonstrated to be key in the strength and frequency of the resulting oscillations in the energy.

(4) The evolution of cross-helicity is in Figs. 3 and 4. Although the definition of magnetic helicity, $H_{M}=\int \mathbf{B} \cdot(\nabla \times \mathbf{B}) d^{3} x$, given in Ref. [25] does not necessarily satisfy a conservation law [29], it is linked with the so-called " $\alpha$-effect" in dynamos [3,15,29] and hence serves as an indicator of dynamo action, if any. In all cases considered, the kinetic helicity is dominant and cross-helicity is negligible.

(5) In the uniform magnetic field cases, the kinetic helicity exhibits oscillations similar to the ones seen in other quantities. The kinetic and magnetic helicities oscillate and decay 


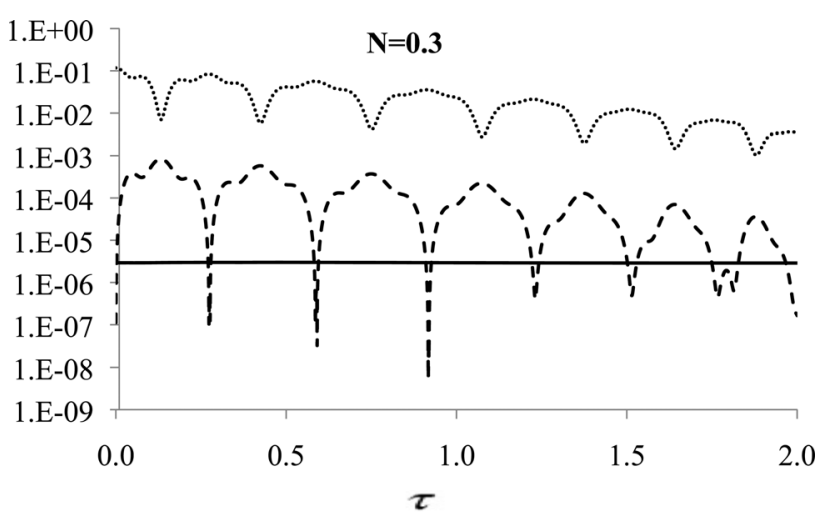

(a)

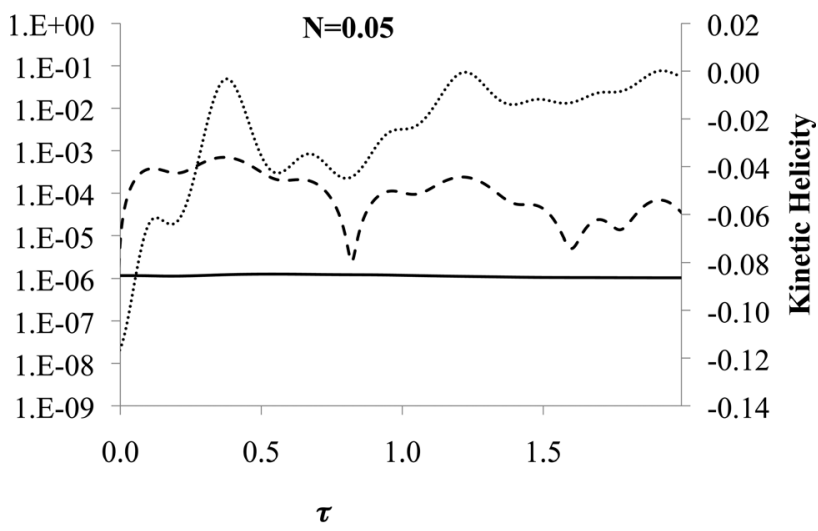

(b)

Fig. 3 Evolution of helicities with $\tau$ (a) $N=0: 3$; (b) $N=0: 05$ (solid: Cross Helicity; ... : Kinetic Helicity, - - - : Magnetic Helicity) [25]

as in the respective forms of energy. The opposite signs indicate the exchange mechanism found in the respective forms of energy. The scaling of helicity with total energy (kinetic and magnetic, $E_{t}=E_{K}+E_{M}$ ) accounts for energy decay. The scaled helicity further unveils the oscillations (Figs. 4(a) and 4(b)) seen in the energy (Fig. 1).

It is well established that the large oscillations in kinetic and magnetic turbulent energies are due to the influence of Alfvén waves propagating through the flow field. This effect manifests via the Lorentz force. However, the details of the interactions between the velocity and magnetic fluctuations under the influence of the Alfvén waves are not quantitatively established. Such quantification is critical for the development of turbulence feedback control strategies and closure modeling. The present work addresses this gap in literature. In this section, we present the dynamic analysis and modeling of the observed simulation results. The quantities of interest are the turbulent kinetic energy, $E_{K}$, magnetic energy, $E_{M}$, and the Lorentz work $E_{L}$. As the turbulence field is homogeneous, these averages are only functions of time and not space.

3.1 Energy Evolution Equations. Here, we briefly present the various steps involved in deriving the evolution equations of turbulent kinetic and magnetic energies. During averaging, we invoke the following:

(1) Mass conservation: $v_{i, i}^{\prime}=0$.

(2) Solenoidal magnetic field (divergence free): $B_{i, i}^{\prime}=0$.

(3) Homogenous turbulence and magnetic field (spatial derivative of volume averaged terms is zero): $(\bar{A})_{, i}=(\bar{A})_{, i}=0$.

We start with writing the Lorentz work term in Eqs. (10) and (11) in indicial notation prior to averaging:

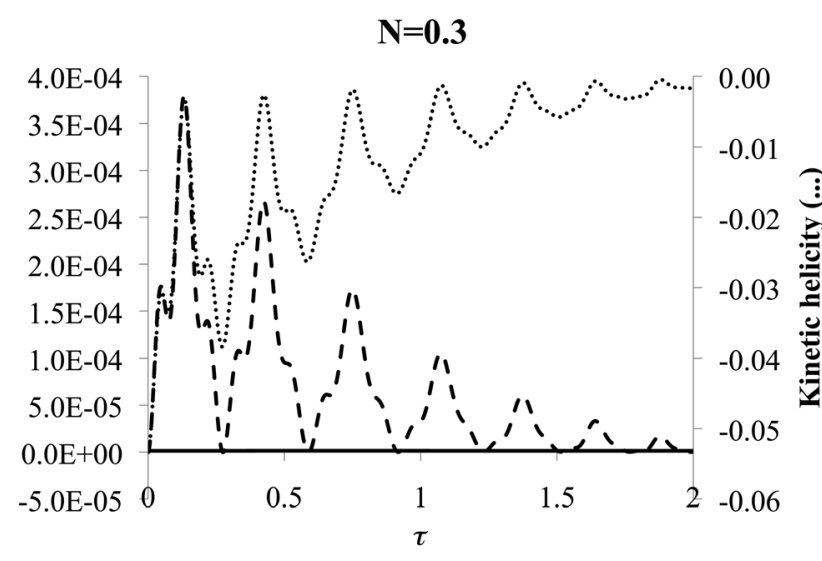

(a)

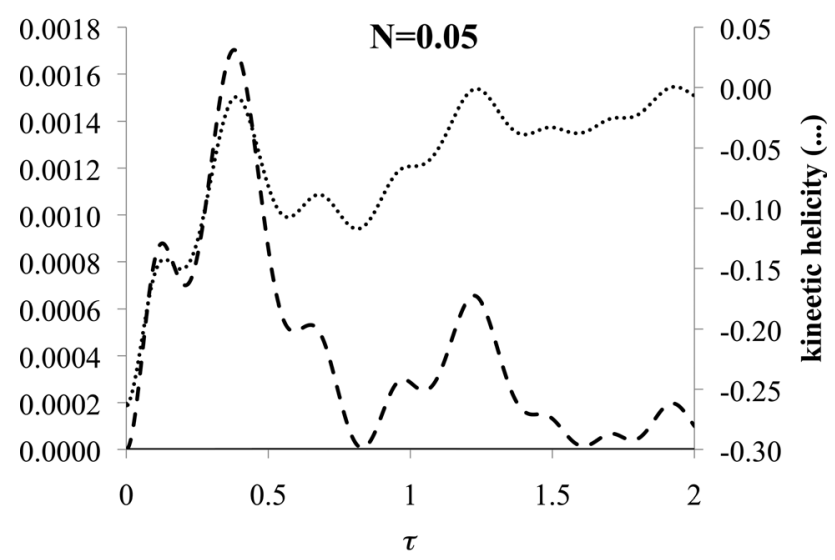

(b)

Fig. 4 Evolution of helicities (scaled to kinetic plus magnetic energy) with $\tau$. (a) $N=0: 3$; (b) $N=0: 05$ (solid: Cross Helicity; ... : Kinetic Helicity, - - - : Magnetic Helicity) [25]

$\mathbf{v}^{\prime} \cdot\left(\mathbf{j}^{\prime} \times \mathbf{B}^{\prime}\right)=\frac{1}{\mu_{0}} \mathbf{v}^{\prime} \cdot\left(\left(\nabla \times \mathbf{B}^{\prime}\right) \times \mathbf{B}^{\prime}\right)=\frac{-1}{\mu_{0}}\left(v_{i}^{\prime} B_{k, i}^{\prime} B_{k}^{\prime}-v_{i}^{\prime} B_{i, k}^{\prime} B_{k}^{\prime}\right)$

Volume-averaging the Lorentz work term gives

$$
\overline{\mathbf{v}^{\prime} \cdot\left(\mathbf{j}^{\prime} \times \mathbf{B}^{\prime}\right)}=-\frac{1}{\mu_{0}}\left(\overline{v_{i}^{\prime} B_{k, i}^{\prime} B_{k}^{\prime}}-\overline{v_{i}^{\prime} B_{i, k}^{\prime} B_{k}^{\prime}}\right)
$$

Consider the term $\overline{v_{i}^{\prime} B_{k, i}^{\prime} B_{k}^{\prime}}$ in Eq. (14)

$$
\begin{aligned}
\overline{v_{i}^{\prime} B_{k, i}^{\prime} B_{k}^{\prime}} & =\overline{\left(v_{i}^{\prime} B_{k}^{\prime} B_{k}^{\prime}\right)_{, i}-v_{i, i}^{\prime} B_{k}^{\prime} B_{k}^{\prime}-v_{i}^{\prime} B_{k}^{\prime} B_{k, i}^{\prime}} \\
& =\left(\overline{v_{i}^{\prime} B_{k}^{\prime} B_{k}^{\prime}}\right)_{, i}-\overline{v_{i, i}^{\prime} B_{k}^{\prime} B_{k}^{\prime}}-\overline{v_{i}^{\prime} B_{k}^{\prime} B_{k, i}^{\prime}} \\
& =0-0-\overline{v_{i}^{\prime} B_{k}^{\prime} B_{k, i}^{\prime}}
\end{aligned}
$$

Similarly,

$$
\begin{aligned}
\overline{v_{i}^{\prime} B_{i, k}^{\prime} B_{k}^{\prime}} & =\overline{\left(v_{i}^{\prime} B_{i}^{\prime} B_{k}^{\prime}\right)_{, k}-v_{i, k}^{\prime} B_{i}^{\prime} B_{k}^{\prime}-v_{i}^{\prime} B_{i}^{\prime} B_{k}^{\prime}, k} \\
& \left.=\overline{\left(v_{i}^{\prime} B_{i}^{\prime} B_{k}^{\prime}\right.}\right)_{, k}-\overline{v_{i, k}^{\prime} B_{i}^{\prime} B_{k}^{\prime}}-\overline{v_{i}^{\prime} B_{i}^{\prime} B_{k}^{\prime}, k} \\
& =0-\overline{v_{i, k}^{\prime} B_{i}^{\prime} B_{k}^{\prime}}-0=-\overline{v_{i, k}^{\prime} B_{i}^{\prime} B_{k}^{\prime}}
\end{aligned}
$$

Thus, the final expression for the volume averaged Lorentz work term is

$$
\overline{\mathbf{v}^{\prime} \cdot\left(\mathbf{j}^{\prime} \times \mathbf{B}^{\prime}\right)}=-\frac{1}{\mu_{0}} \overline{\mathbf{B}^{\prime} \cdot\left(\left(\mathbf{B}^{\prime} \cdot \nabla\right) \mathbf{v}^{\prime}\right)}
$$


Consider the first term on the RHS (which is the divergence of the Poynting vector) in the instantaneous magnetic energy equation, Eq. (11). Using Eq. (12) and noting that $\left(\mathbf{v}^{\prime} \times \mathbf{B}^{\prime}\right) \times \mathbf{B}^{\prime}=\mathbf{v}^{\prime} \times\left(\mathbf{B}^{\prime} \times \mathbf{B}^{\prime}\right)=\mathbf{0}$,

$$
\begin{aligned}
& -\frac{1}{\mu_{0}} \nabla \cdot\left(\mathbf{E}^{\prime} \times \mathbf{B}^{\prime}\right)=-\frac{1}{\sigma \mu_{0}} \nabla \cdot\left(\mathbf{j}^{\prime} \times \mathbf{B}^{\prime}\right) \\
= & \frac{\eta}{\mu_{0}}\left(B_{k, i}^{\prime} B_{k, i}^{\prime}+B_{k}^{\prime} B_{k, i i}^{\prime}-B_{k, i}^{\prime} B_{i, k}^{\prime}-B_{k}^{\prime} B_{i, k i}^{\prime}\right)
\end{aligned}
$$

Next, consider the Joule dissipation term on the RHS of the instantaneous magnetic energy equation, Eq. (11),

$$
\eta \mu_{0} j^{\prime 2}=\frac{\eta}{\mu_{0}}\left(\nabla \times \mathbf{B}^{\prime}\right) \cdot\left(\nabla \times \mathbf{B}^{\prime}\right)=\frac{\eta}{\mu_{0}}\left(-B_{k, i}^{\prime} B_{k, i}^{\prime}+B_{k, i}^{\prime} B_{i, k}^{\prime}\right)
$$

Combining Eqs. (18) and (20) we get

$$
\frac{-\nabla \cdot\left(\mathbf{E}^{\prime} \times \mathbf{B}^{\prime}\right)}{\mu_{0}}-\eta \mu_{0} j^{\prime 2}=\frac{\eta}{\mu_{0}}\left(\left(B_{k}^{\prime} B_{k, i}^{\prime}\right)_{, i}-B_{k, i}^{\prime} B_{k, i}^{\prime}\right)
$$

Averaging gives

$$
\overline{-\frac{1}{\mu_{0}} \nabla \cdot\left(\mathbf{E}^{\prime} \times \mathbf{B}^{\prime}\right)-\eta \mu_{0} j^{2}}=-\frac{\eta}{\mu_{0}}\left(\overline{B_{k, i}^{\prime} B_{k, i}^{\prime}}\right)
$$

The turbulent kinetic and magnetic energies,

$$
E_{K}=\rho \overline{v_{i}^{\prime} v_{i}^{\prime}} / 2, \quad E_{M}=\overline{B_{i}^{\prime} B_{i}^{\prime}} / 2 \mu_{0}
$$

evolve as

$$
\begin{gathered}
\frac{d E_{K}}{d t}=-\mu \overline{\left(v_{k, i}^{\prime} v_{k, i}^{\prime}\right)}-\frac{1}{\mu_{0}}\left(\overline{v_{i, k}^{\prime} B_{i}^{\prime} B_{k}^{\prime}}\right) \\
\frac{d E_{M}}{d t}=-\frac{\eta}{\mu_{0}}\left(\overline{B_{k, i}^{\prime} B_{k, i}^{\prime}}\right)+\frac{1}{\mu_{0}}\left(\overline{v_{i, k}^{\prime} B_{i}^{\prime} B_{k}^{\prime}}\right)
\end{gathered}
$$

The two equations are quite similar in form and contain dissipation terms. The second term (Lorentz work term) is the same in both equations, but appears with opposite signs. This is the coupling term which is responsible for the transfer of energy from the kinetic to magnetic mode as noted with Eqs. (10) and (11). Summing Eqs. (23) and (24), the resulting equation for the total energy is

$$
\frac{d E_{t}}{d t}=-\mu\left(\overline{v_{k, i}^{\prime} v_{k, i}^{\prime}}\right)-\frac{\eta}{\mu_{0}}\left(\overline{B_{k, i}^{\prime} B_{k, i}^{\prime}}\right)
$$

While the evolution of total energy is simple monotonic decay [14], the partition and interchange between kinetic and magnetic energies is clearly more complex.

3.2 Modeling the Energy Exchange. The evolution of the kinetic, magnetic, total energy, and the Lorentz work is shown as a function of the eddy turnover time $\left(\tau=t E_{k} / \varepsilon\right.$, where $\left.\varepsilon=\mu \overline{\left(v_{k, i}^{\prime} v_{k, i}^{\prime}\right)}\right)$, in Fig. 2(a) for two values of the interaction parameter $N$. The total energy

$$
E_{t}=E_{K}+E_{M}
$$

seems to decay exponentially similarly to other quantities observed in turbulence $[7,8,14]$. Therefore we postulate the following decay law

$$
\frac{d E_{t}}{d t}=-\Lambda E_{t}
$$

with solution ( $E_{t}^{0}$ being the initial value of the total energy)

$$
E_{t}=E_{t}^{0} e^{-\Lambda t}
$$

The exponential decay model for the total energy requires further discussion. It is well-known that in fluid (non-MHD) turbulence, the kinetic energy experiences power-law decay. In Ref. [25], it is seen that there are important differences in kinetic energy decay due to the presence of a magnetic field. The results shown here support an exponential decay model for the total energy.

Equation (25) also suggests

$$
E_{K} \propto \mu\left(\overline{v_{k, i}^{\prime} v_{k, i}^{\prime}}\right), \quad E_{M} \propto \frac{\eta}{\mu_{0}}\left(\overline{B_{k, i}^{\prime} B_{k, i}^{\prime}}\right)
$$

The data for the $N=0.3$ case (Fig. $2(a)$ ) yield $E_{t}^{0} \simeq 0.11$ and $\Lambda \simeq 1.2$.

The exponential decay is easily removed from the kinetic and magnetic energy plots by normalization with the total energy. The normalized kinetic and magnetic energy (Fig. 5) are symmetric about the line $E=0.5$. We conclude that, at times, all energy is present in the kinetic mode. Then the action of the Lorentz work transfers energy to the magnetic mode and very quickly energy is equipartitioned between the kinetic and magnetic modes. After a few oscillations, the Lorentz work again deposits all energy in the kinetic mode and the pattern repeats. The difference between successive oscillations is the damping of the intermittent oscillations to an extent that oscillations are totally dissipated.

The Fourier spectrum of the normalized kinetic energy reveals three peaks at frequencies

$$
\omega_{1}=11, \quad \omega_{2}=22, \quad \omega_{3}=33
$$

Note that the frequencies are commensurate, with ratio of $\omega_{1}: \omega_{2}: \omega_{3}=1: 2: 3$.

While the amplitudes corresponding to different frequencies are not the same, the simplest model represents $E_{K}$ and $E_{M}$ as sums of three modes

$$
E_{K}=\sum_{j=1}^{3} E_{K j}, \quad E_{M}=\sum_{j=1}^{3} E_{M j}
$$

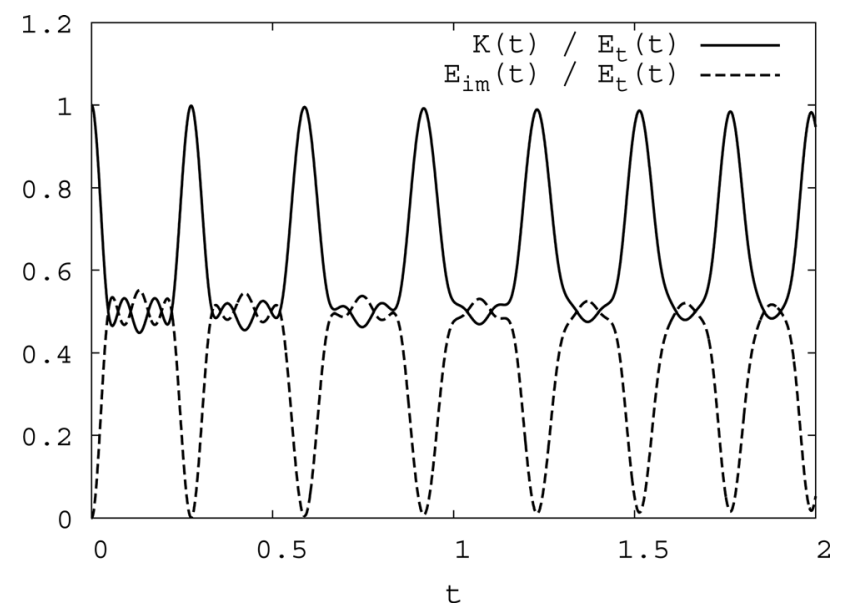

Fig. 5 Kinetic and magnetic energy (normalized with total energy) vs. eddy turnover time 
We now posit the following simple phenomenological model for the kinetic energy

$$
\begin{gathered}
E_{K j}=\frac{E_{t}^{0}}{2} e^{-\Lambda t}\left(1+\cos ^{2}\left(\omega_{j} t\right)\right) \\
E_{K}=\frac{E_{t}^{0}}{2} e^{-\Lambda t} \sum_{j=1}^{3}\left(1+\cos ^{2}\left(\omega_{j} t\right)\right)
\end{gathered}
$$

and a corresponding expression for the magnetic energy as

$$
E_{M j}=\frac{E_{t}^{0}}{2} e^{-\Lambda t} \sin ^{2}\left(\omega_{j} t\right), \quad E_{M}=\frac{E_{t}^{0}}{2} e^{-\Lambda t} \sum_{j=1}^{3} \sin ^{2}\left(\omega_{j} t\right)
$$

Both energies are nonnegative and the total energy then decays exponentially as required by Eq. (27).

However, the amplitudes corresponding to different frequencies are not the same. This suggests the following model representation:

$$
\begin{aligned}
E_{K} & =\frac{E_{t}^{0}}{c_{1}+c_{2}+c_{3}} e^{-\Lambda t} \sum_{j=1}^{3}\left(c_{j} \cos ^{2}\left(\omega_{j} t\right)+\frac{\Delta}{3}\right) \\
& =\frac{E_{t}^{0} e^{-\Lambda t}}{2 \sum_{j=1}^{3} c_{j}} \sum_{j=1}^{3}\left(c_{j}\left(1+\cos \left(2 \omega_{j} t\right)\right)+\frac{2 \Delta}{3}\right)
\end{aligned}
$$

and similarly

$$
\begin{aligned}
E_{K} & =\frac{E_{t}^{0}}{c_{1}+c_{2}+c_{3}} e^{-\Lambda t} \sum_{j=1}^{3}\left(c_{j} \sin ^{2}\left(\omega_{j} t\right)\right) \\
& =\frac{E_{t}^{0} e^{-\Lambda t}}{2 \sum_{j=1}^{3} c_{j}} \sum_{j=1}^{3}\left(c_{j}\left(1+\cos \left(2 \omega_{j} t\right)\right)\right)
\end{aligned}
$$

Note that

$$
E_{K j}+E_{M j}=\frac{E_{t}^{0}}{\sum_{j=1}^{3} c_{j}} e^{-\Lambda t}\left(c_{j}+\frac{\Delta}{3}\right)
$$

which enables specifying the initial conditions for each $j=1,2,3$ as

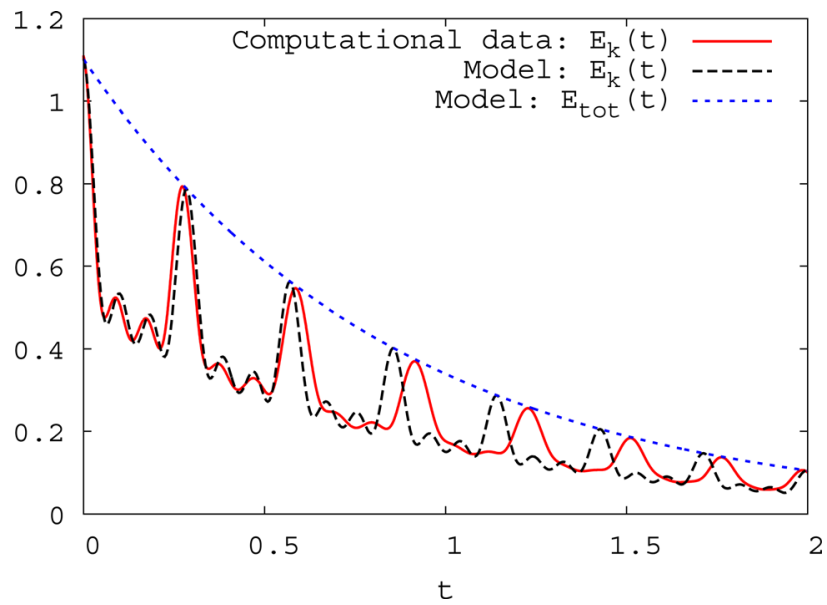

Fig. 6 Comparison of computational data with proposed model

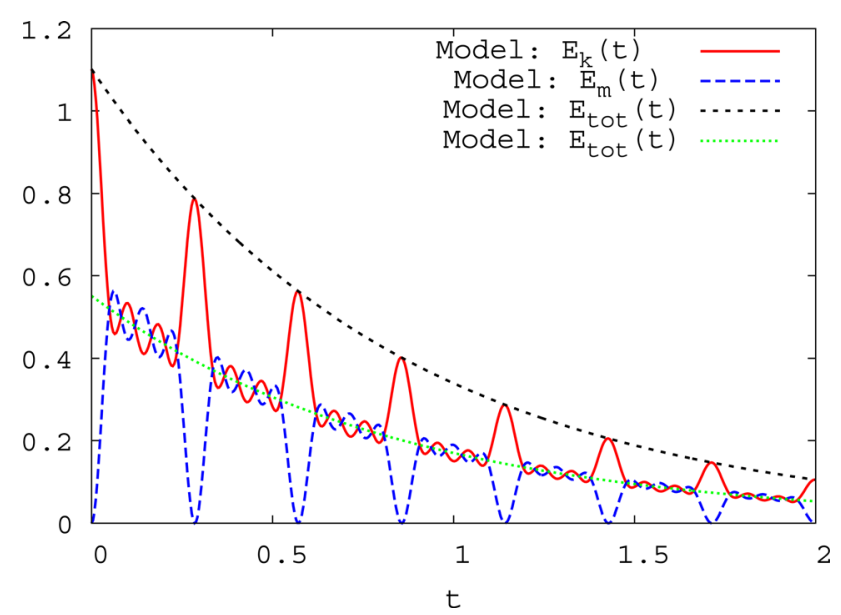

Fig. 7 Kinetic and Magnetic Energy plot from the model

$$
E_{M j}^{0}=0, \quad E_{K j}^{0}=\frac{E_{t}^{0}}{2 \sum_{j=1}^{3} c_{j}}\left(c_{j}+\frac{\Delta}{3}\right)
$$

The above model also verifies the postulate in Eq. (18).

From Fourier analysis we get

$$
\begin{gathered}
c_{1}^{2}=15, \quad c_{2}^{2}=8, \quad c_{3}^{2}=5 \\
c_{1}=3.873, \quad c_{2}=2.828, \quad c_{3}=2.236
\end{gathered}
$$

Figures 6 and 7 show the comparisons of the model with the computational results. The model matches the data for first two peaks very well. The lag after the first two peaks can be attributed to the omitted phase shifts and nonlinear changes in the period.

Now, based on the improved model we want to quantify the equipartition of energy. The data supports the statement that at any given instant, kinetic and magnetic energy are equally spaced on either side of half of the total energy, i.e.,

$$
E_{K}=\frac{E_{t}}{2}+\zeta \quad \text { and } \quad E_{K}=\frac{E_{t}}{2}-\zeta
$$

Thus

$$
\zeta=\frac{E_{K}-E_{M}}{2}=\frac{c e^{-\Lambda t}}{2 \sum_{j=1}^{3} c_{j}} \sum_{j=1}^{3}\left(c_{j} \cos \left(2 \omega_{j} t\right)\right)+\frac{c e^{-\Lambda t}}{2 \sum_{j=1}^{3} c_{j}} \Delta
$$

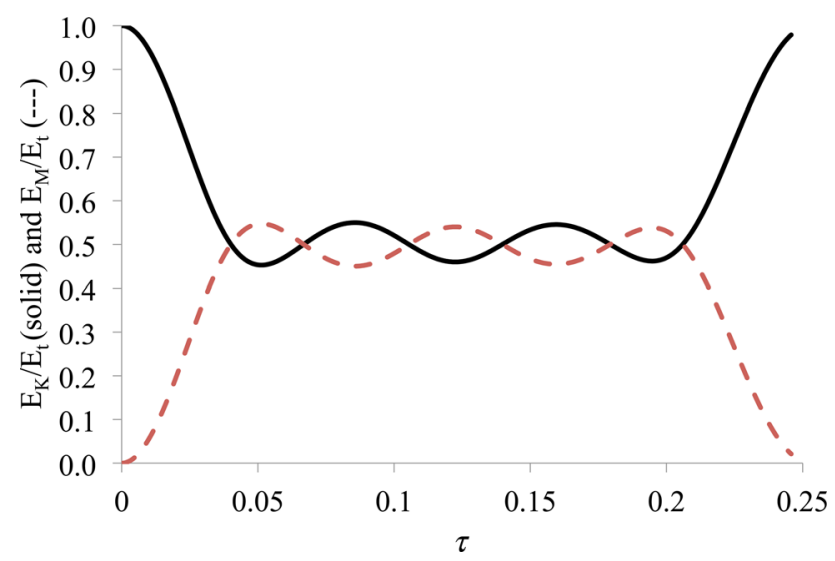

Fig. 8 Evolution of $E_{M} / E_{t}$ and $E_{K} / E_{t}$ with $\tau$. (solid:Kinetic Energy; - - - : Magnetic Energy) 
This leads us to the following final observation. Although there is a continuous exchange of energy between the kinetic and magnetic mode, the energy is not equally partitioned. There is a part of kinetic energy that is not exchanged. The percentage of un-exchanged energy is given by

$$
\frac{\frac{c}{\sum_{j=1}^{3} c_{j} e^{-\Lambda t} \Delta}}{\frac{c}{\sum_{j=1}^{3} c_{j}} e^{-\Lambda t}\left(\sum_{j=1}^{3} c_{j}+\Delta\right)}=\frac{\Delta}{\sum_{j=1}^{3} c_{j}+\Delta}
$$

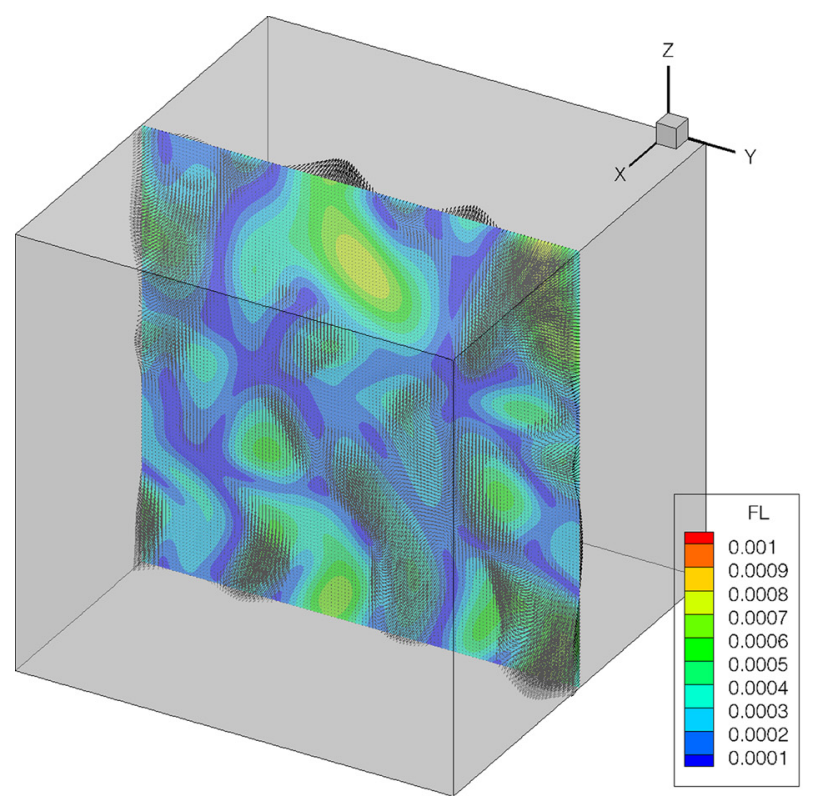

(a)

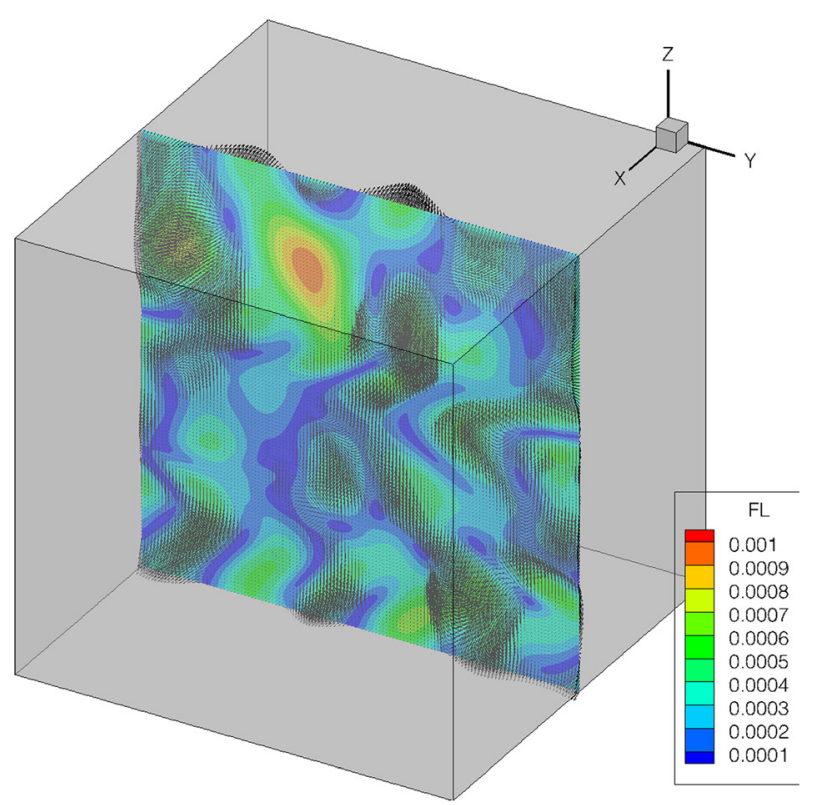

(c)
This indicates as much as $31 \%$ of the energy is not exchanged for the $N=0.3$ case

\section{Dynamics and Kinematics of Energy Exchange}

It is necessary to closely examine the kinetic and magnetic energies to better identify the cause and effect relationship of the dynamics in MHD turbulence in a uniform magnetic field. We now look more closely at the fields and their variations in a specific time range.

The interaction between the velocity and magnetic energies is brought about by Lorentz work as previously shown [25]. In Fig. 2, the evolution of Lorentz work is shown and the two

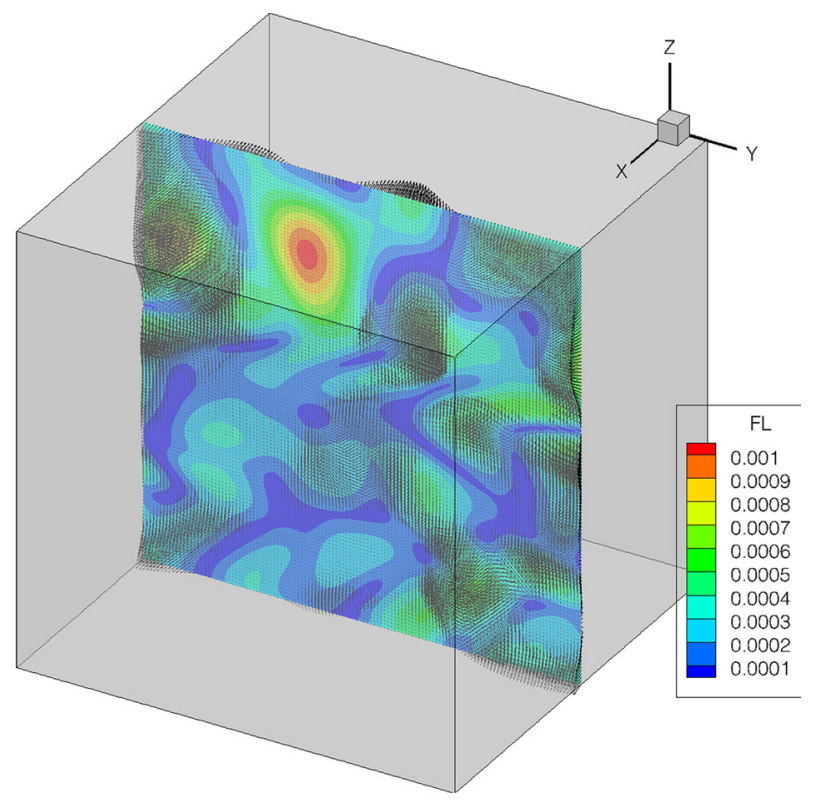

(b)

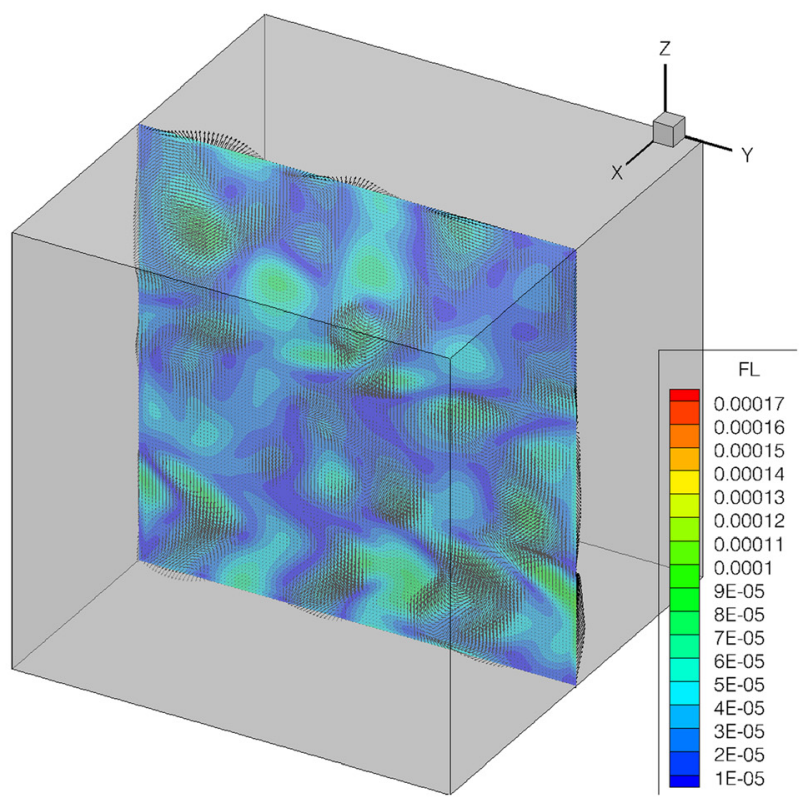

(d)

Fig. 9 The Lorentz force at (a) $\tau=0: 12$, (b) $\tau=0: 18$, (c) $\tau=0: 2$ and (d) $\tau=0: 25$ 
energies are also presented to demonstrate the exchanges clearly. Note the nonmonotonic approach to equipartition in the cases with a mean magnetic field applied. To examine the exchange between kinetic and magnetic energies without the complications of dissipation, we present the individual energies normalized by the total energy at that time instance in Fig. 8. The figure covers a time period $\tau=(0,0.25)$ which represents the first full wavelength of oscillatory exchange. That pattern repeats itself with

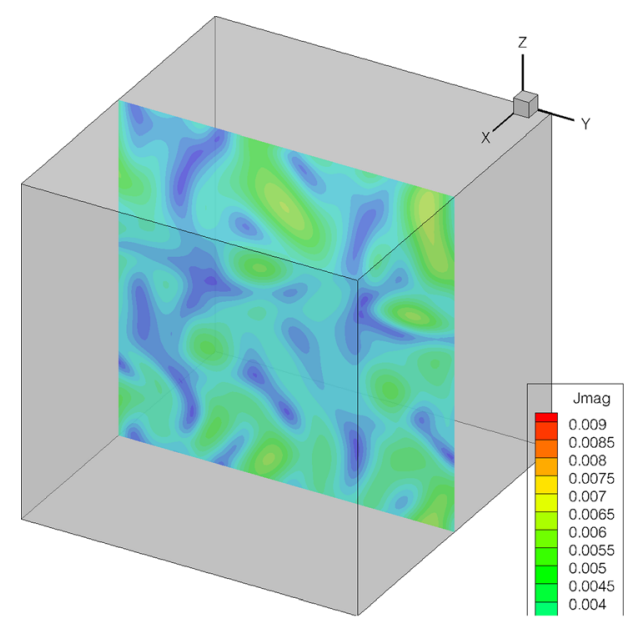

(a)

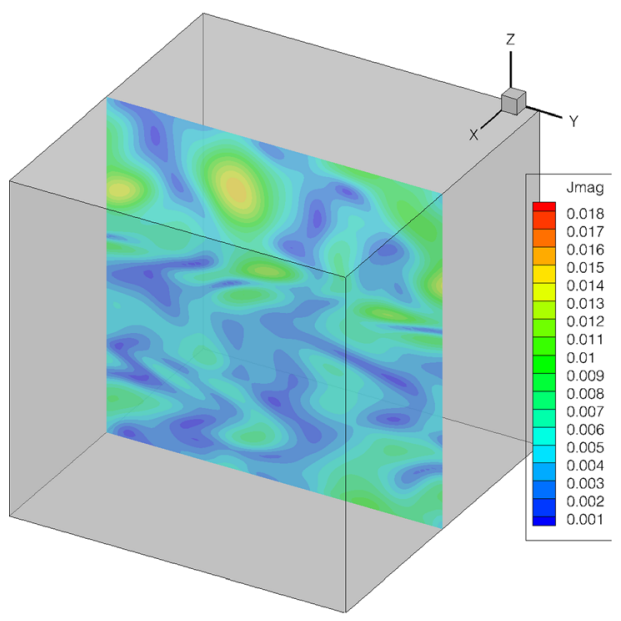

(c)

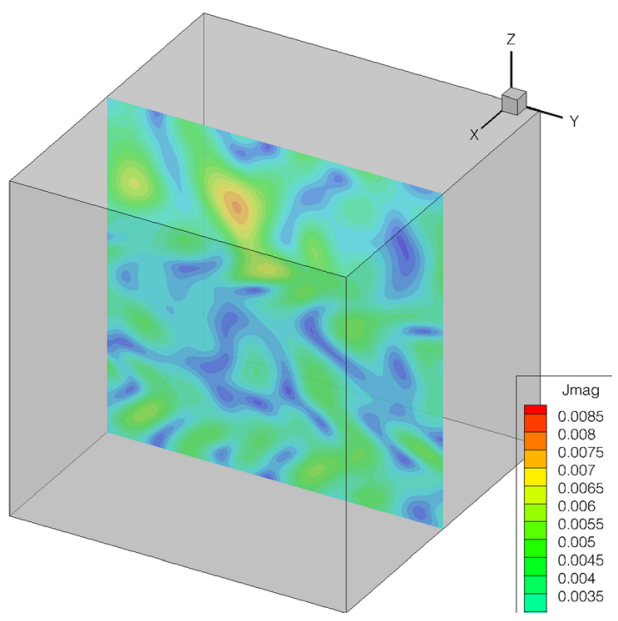

(e)

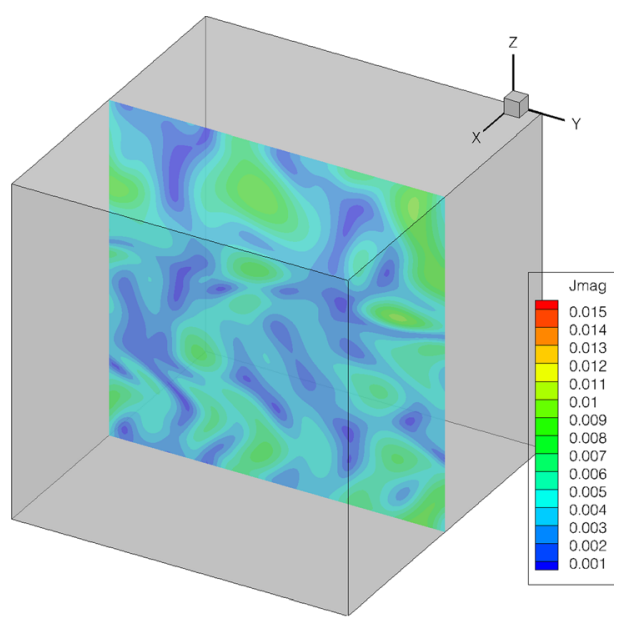

(b)

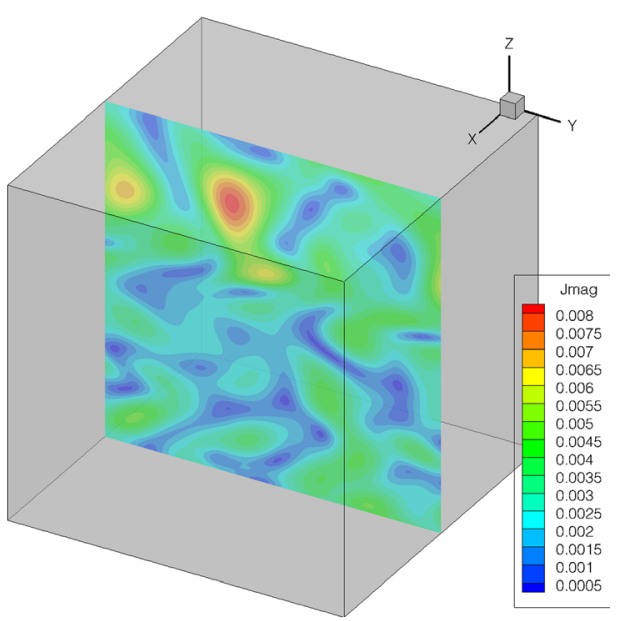

(d)

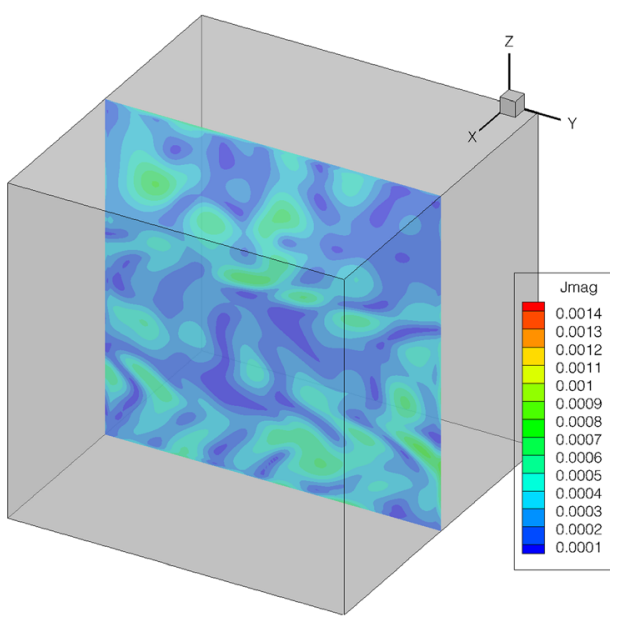

(f)

Fig. 10 Current density progression indicating propagation of Alfvén wavesas also seen in other studies [1] (a) $\tau=0: 12$, (b) $\tau=0: 138$, (c) $\tau=0: 158$, (d) $\tau=0: 18$, (d) $\tau=0: 2$ and (e) $\tau=0: 25$ 


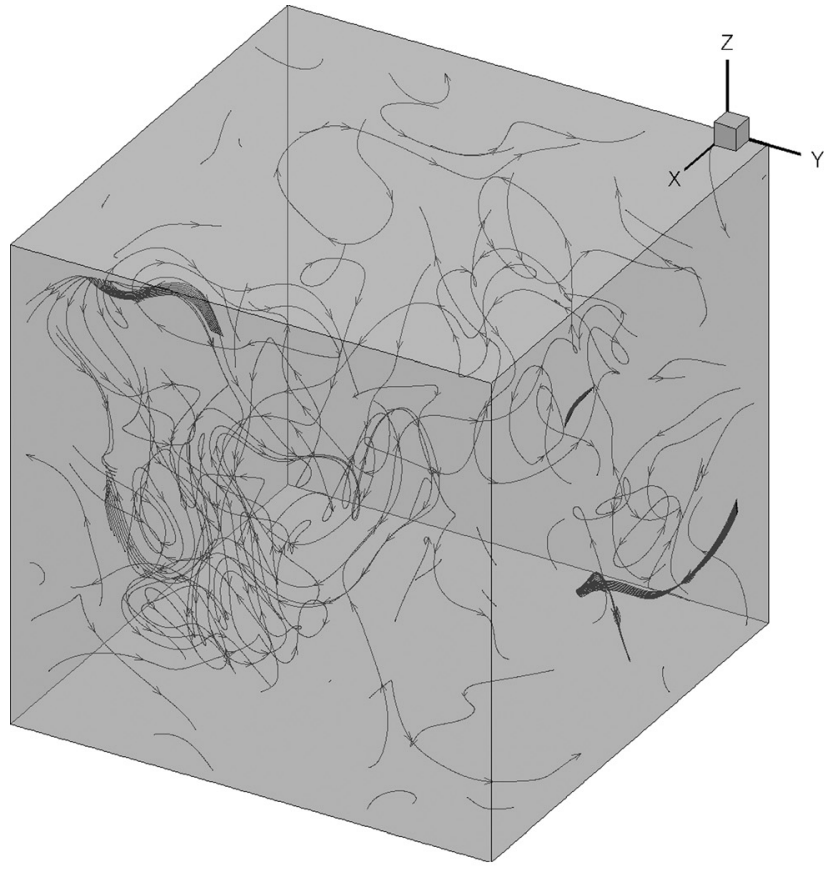

(a)

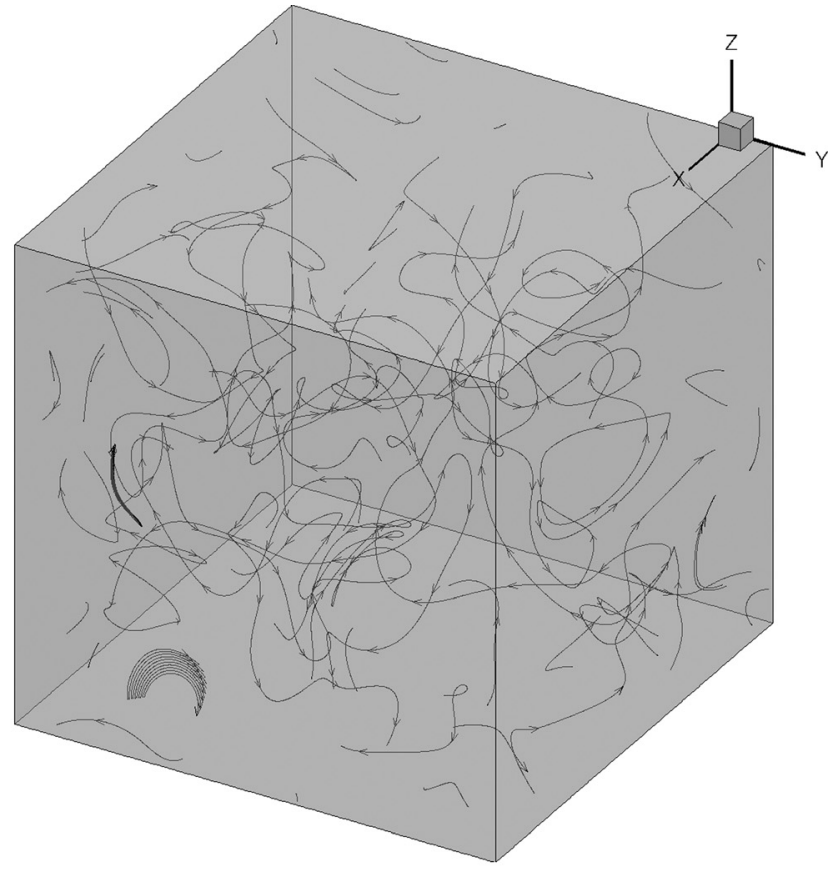

(b)

Fig. 11 Streamlines at eddy turnover times, (a) $\tau=0.18$ and (b) $\tau=0.25$

minor modifications at later times. Much of the physics of interest to this study is indeed resident in the time period $\tau=0$ to $\tau=0.25$.

We closely examine the behavior of key variables at the peaks and troughs in the oscillations. The times at which the peaks and troughs occur are at the following approximate eddy turnover times: $\tau=0.12,0.18,0.2$, and 0.25 . These are times when $E_{K}$ and $E_{M}$ reach extremum values. For example, $E_{M}(\tau=0.12)$ is a maximum of magnetic energy while $E_{M}(\tau=0.25)$ is a minimum of magnetic energy (other than the initial conditions). Conversely,

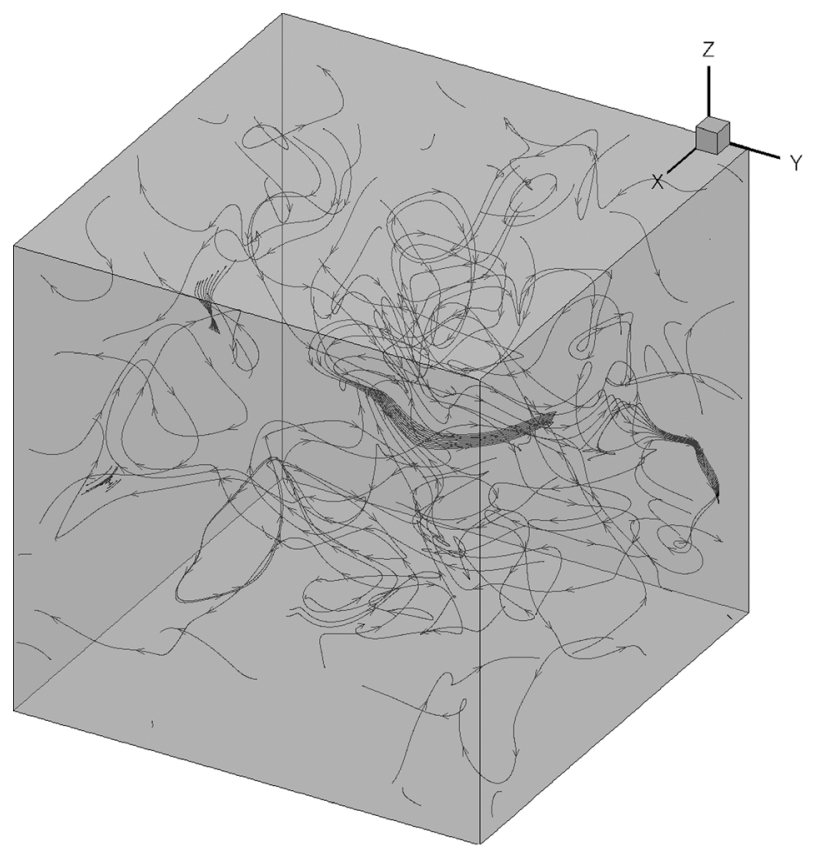

(a)

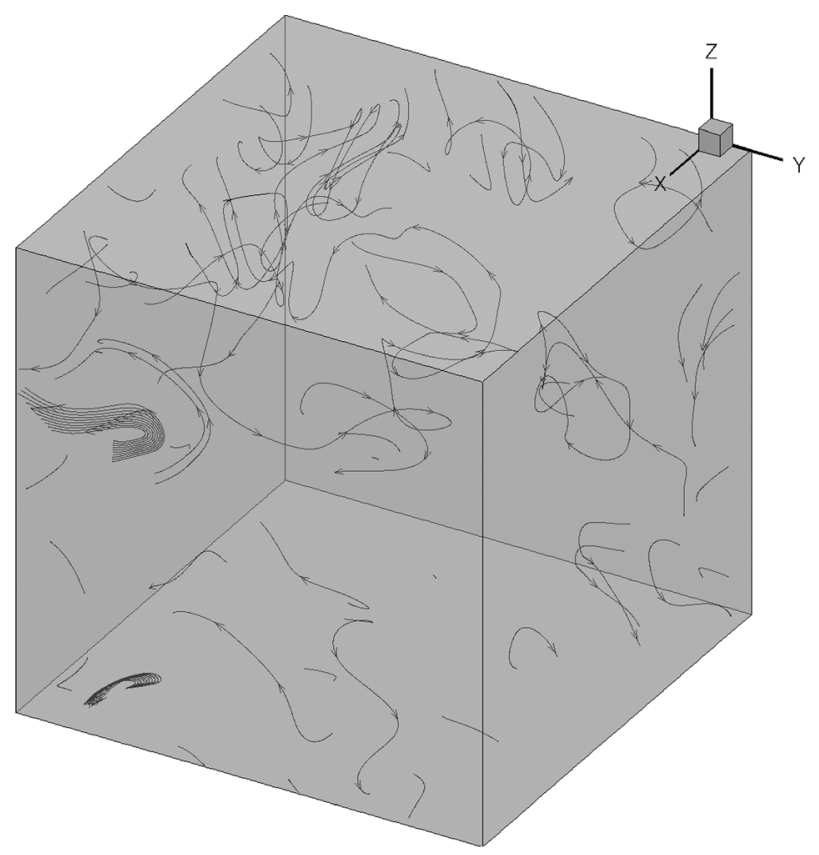

(b)

Fig. 12 Vorticity field "streamlines" at eddy turnover times, (a) $\tau=0.18$ and (b) $\tau=0.25$ 


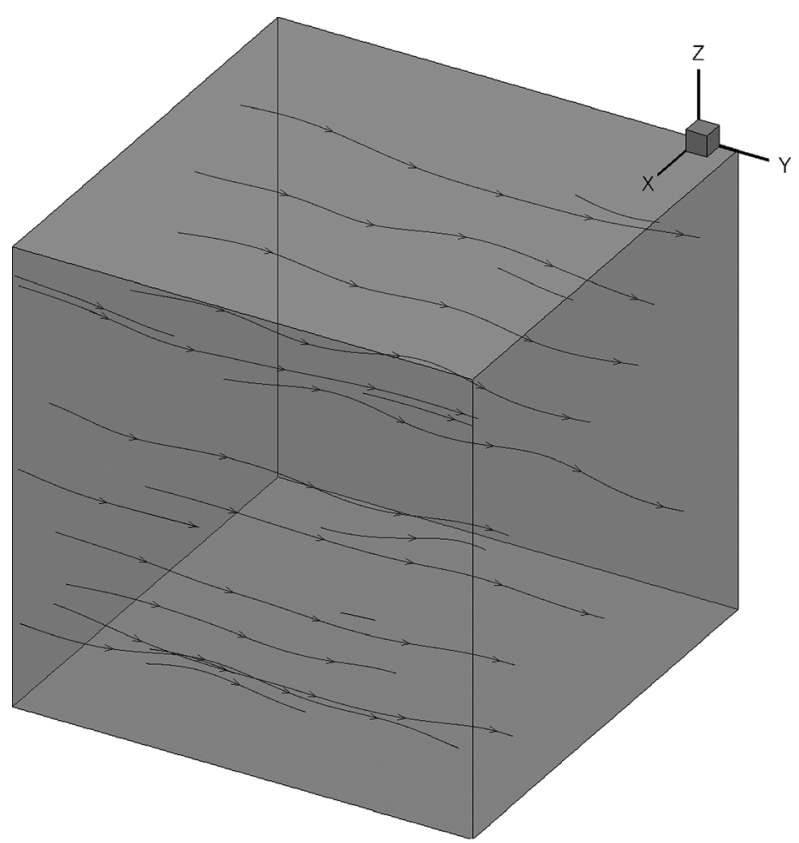

(a)

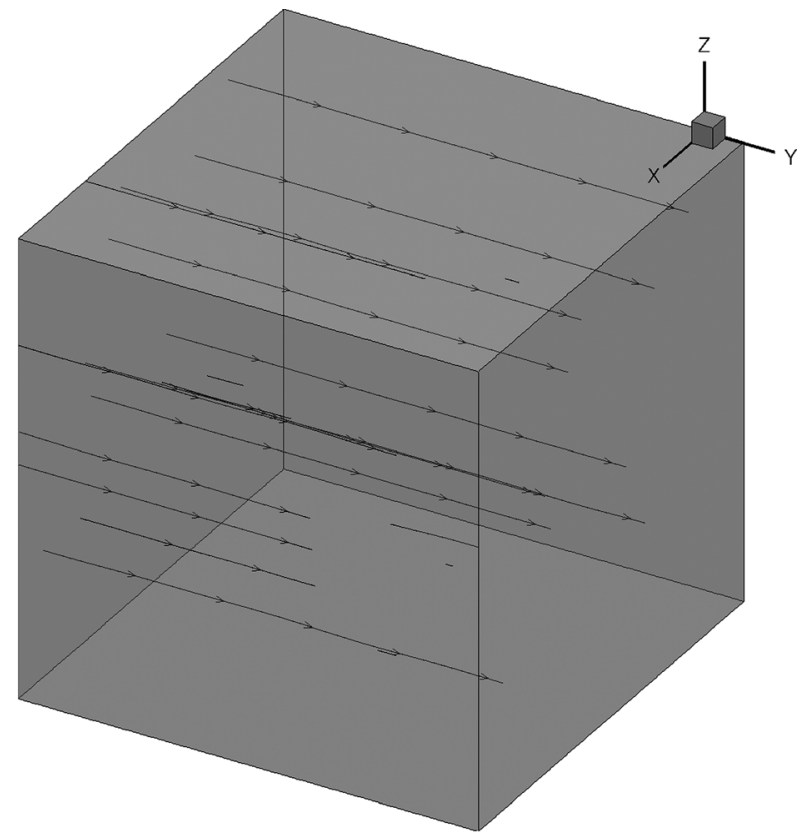

(b)

Fig. 13 Magnetic field "streamlines" at eddy turnover times, (a) $\tau=0.18$ and (b) $\tau=0.25$

the $\tau=0.12$ and 0.25 are times that correspond to a minimum and a maximum of kinetic energy, respectively. The $\tau=0.12$ and 0.25 are also times when the rate of change of kinetic and magnetic energies vanish. The $\tau=0.2$ is a "crossover" time where the magnetic and kinetic energy curves cross: $E_{M}=E_{K}$.

Now we present observations regarding the helicities of the cases in Figs. 3(a)-4(b). In Fig. 3(a), at $\tau=0.12$, there is a trough in the kinetic helicity in correspondence with the kinetic energy. Similarly, there is a peak at 0.18 in both kinetic and magnetic helicity. After this time, the frequency of oscillation slows down. Note that there are stronger oscillations in the $N=0.3$ case with the stronger magnetic field whereas the oscillations seem to be weaker for the $N=0.05$ case. This is an indication of a greater likelihood of dynamo action at stronger magnetic fields $[3,15,29]$, although none of these cases considered here has sustained helicity large enough to cause such action.

The Lorentz force (vector form) is plotted in Fig. 9. This figure, not only shows the magnitude of the Lorentz force to be lowest when the magnetic energy is lowest at $\tau=0.25$, but also indicates that the Lorentz force switches direction between $\tau=0.12$ and $\tau=0.18$ in some key locations. The Lorentz force vectors are extremely small at $\tau=0.25$ corresponding to the weak Lorentz force. This is coincident with the lowest magnetic energy at $\tau=0.25$ as seen in Fig. 8. The changes observed at these different times are interpreted as passing of Alfvén waves [1,54-56]. We plot the current density magnitude contours in Fig. 10 to show Alfvén wave propagation as shown in other studies [1,54-56]. The time intervals yield the frequency observed in the nonlinear dynamical systems analysis in the previous section.

The alignment of the velocity with the Lorentz force, i.e., the alignment of the effect with the cause is given by:

$$
\cos (\Theta)=\frac{\overline{\mathbf{v}^{\prime} \cdot\left(\mathbf{j}^{\prime} \times \mathbf{B}^{\prime}\right)}}{\sqrt{\overline{\mathbf{v}^{\prime 2}}} \sqrt{\overline{\overline{\left(\mathbf{j}^{\prime} \times \mathbf{B}^{\prime}\right)^{2}}}}}
$$

This average value is nearly 0 at all times, indicating no preferential statistical alignment between the velocity field and the Lorentz force. The velocity and magnetic fields suggest local, if not global, alignments according to the times and locations where they have their extrema. Figures 11-14 show streamlines, vectors or contours of velocity, vorticity or magnetic induction fields at times that coincide with maxima and minima of energy in Fig. 8.

(1) Figure 11(a) shows the streamlines more clustered at $\tau=0.18$. This is when Fig. 8 shows the magnetic energy is crossing the kinetic energy as it rises to a peak. Figure $11(b)$ shows the streamlines more dispersed at $\tau=0.25$, which is when Fig. 8 indicates that the kinetic energy is highest and the magnetic energy is lowest.

(2) Figure 12(a) shows the equivalent streamlines of the vorticity field at $\tau=0.18$, when the lines are more clustered. Figure $12(b)$ shows the vorticity streamlines at $\tau=0.25$, when the lines are more dispersed which is when the kinetic energy is highest.

(3) Figure 13(a) shows the equivalent streamlines of the magnetic field with a lot of fluctuations. Figure 13(b) shows the dominance of the mean magnetic field over the fluctuations at $\tau=0.25$.

The large change in these streamlines from $\tau=0.2$ to $\tau=0.25$ correspond to high energy rates of change during the oscillations according to the constants in Eq. (36) of the model. These changes observed at these different times are evidence of the noted exchange mechanism due to the Lorentz force. The repetition of these patterns suggests that they are manifestations of Alfvén waves on the streamline fields [57].

The magnitude of the vorticity field, plotted in Fig. 14, shows the contours elongated in the direction of the applied mean magnetic field at $\tau=0.2$ suggesting strong alignment. Figure $14(d)$ shows the highest values of the vorticity corresponding with the highest values of the kinetic energy at $\tau=0.25$ in Fig. 8. These effects on the contours are due to the Lorentz force exchange mechanism and are also indications of Alfvén waves [1,54-56]. 


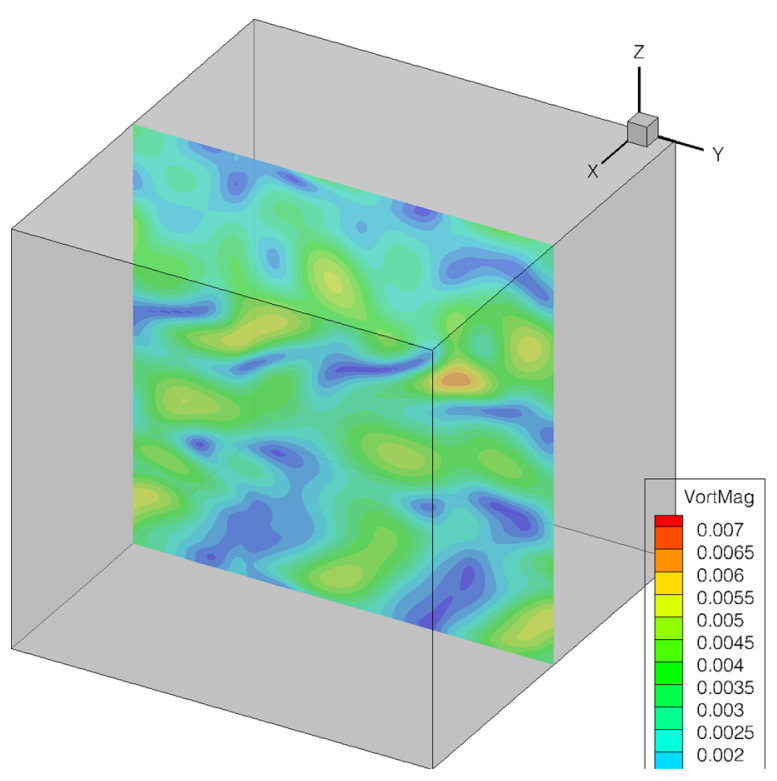

(a)

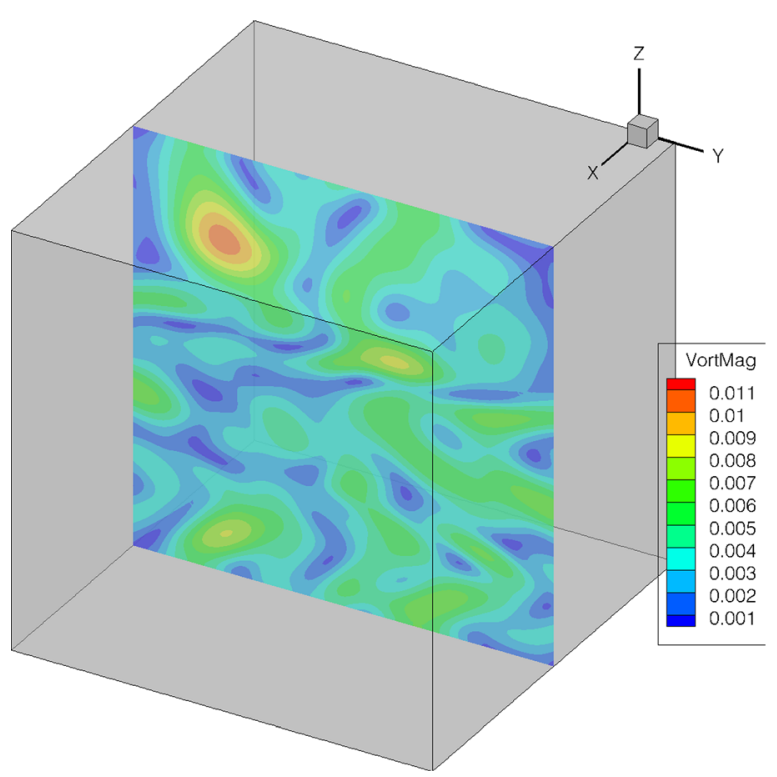

(c)

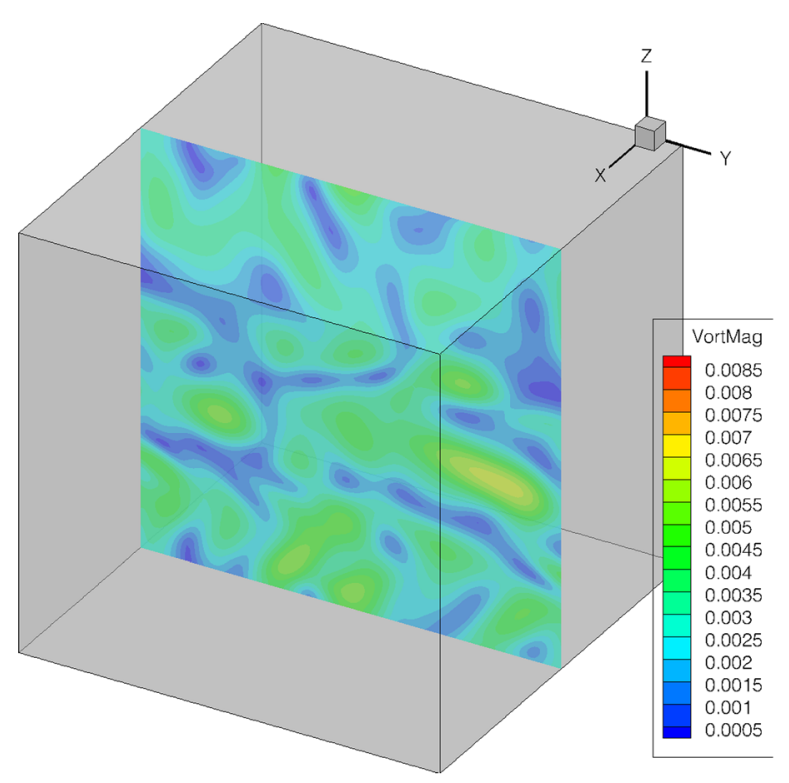

(b)

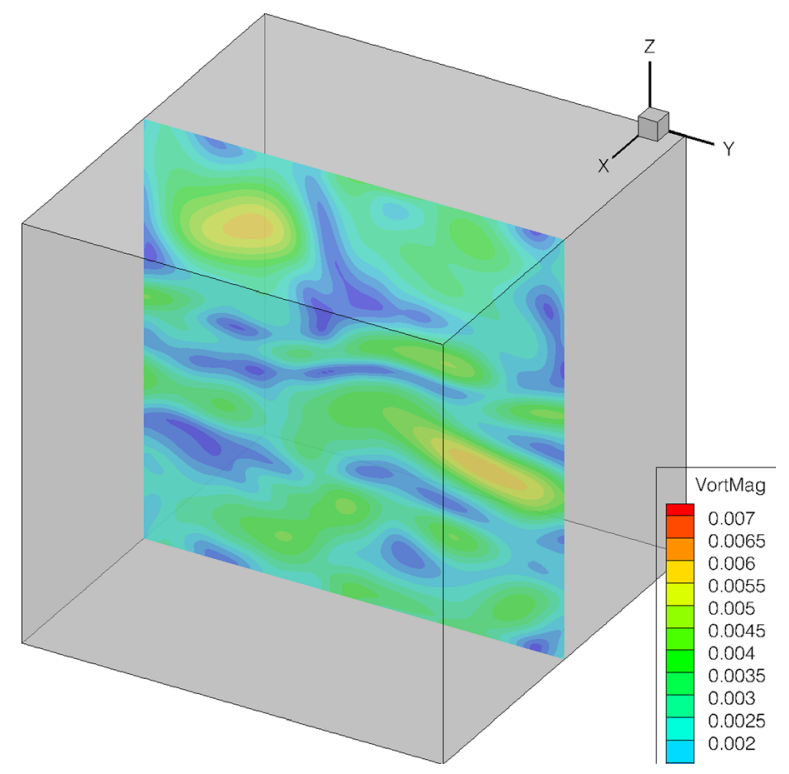

(d)

Fig. 14 Magnitude of the vorticity at (a) $\tau=0: 12$, (b) $\tau=0: 18$, (c) $\tau=0: 2$ and (d) $\tau=0: 25$

\section{Conclusion}

Under the influence of a uniform magnetic field, MHD turbulence decay exhibits complex flow-magnetic field interactions due to the presence of Alfvén waves. The main effect of the Alfvén waves is to cause large oscillations in the turbulent kinetic and magnetic energies even as the two approach equipartition. This is in contrast to a monotonic evolution toward magnetic-kinetic equipartition in the absence of the background field. While the qualitative aspects of the effects of Alfvén waves are well understood, we present for the first time a quantitative analysis of the magnetic-velocity field interactions. Subject to the conditions of this study, the DNS results reveal three dominant frequencies in the kinetic-magnetic energy exchange-corresponding to three Alfvén frequencies. A detailed analysis of the flow-magnetic field coupling is performed and a simple dynamic model is developed to mimic the observed energy exchange. The underlying physics is also examined. Overall, the present work provides important insight required for developing MHD control strategies and turbulence closure models.

\section{Acknowledgment}

Tamás Kalmár-Nagy acknowledges support by the US Air Force Office of Scientific Research under Grant 323 No. FA955008-1-0333.

\section{References}

[1] Alboussière, T., Cardin, P., Debray, F., La Rizza, P., Masson, J., Plunian, F. Ribeiro, A., and Schmitt, D., 2011, "Experimental Evidence of Alfvén Wave Propagation in a Gallium Alloy," Phys. Fluids, 23, p. 096601.

[2] Brandenburg, A., 2003, "Computational Aspects of Astrophysical MHD and Turbulence," Advances in Nonlinear Dynamos, The Fluid Mechanics of 
Astrophysics and Geophysics, Vol. 9, A. M. Soward, C. A. Jones, and D. W. Hughes, eds., CRC Press, Boca Raton, FL, pp. 269-344.

[3] Brandenburg, A., and Käpylä, P., 2007, "Magnetic Helicity Effects in Astrophysical and Laboratory Dynamos," New J. Phys., 9, pp. 305-330.

[4] Mininni, P., Ponty, Y., Montgomery, D., Pinton, J., Politano, H., and Pouquet, A., 2005, "Dynamo Regimes With a Nonhelical Forcing," Astrophys. J., 626, pp. 853-863.

[5] Pouquet, A., and Patterson, G., 1978, "Numerical Simulation of Helical Magnetohydrodynamic Turbulence," J. Fluid Mech., 85(2), pp. 305-323.

[6] Davidson, P. A., 2001, An Introduction to Magnetohydrodynamics, Cambridge University Press, Cambridge, UK

[7] Pope, S., 2000, Turbulent Flows, Cambridge University Press, Cambridge, UK.

[8] Davidson, P., 2004, Turbulence: An Introduction for Scientists and Engineers, Oxford University Press, New York.

[9] Roy, R. I. S., Hastings, D. E., and Taylor, S., 1996, “Three-Dimensional Plasma Particle-in-Cell Calculations of Ion Thruster Backflow Contamination,” J. Comput. Phys., 128, pp. 6-18.

[10] Haas, J. M., and Gallimore, A. D., 2001, "Internal Plasma Potential Profiles in a Laboratory-Model Hall Thruster," Phys. Plasma, 38(2), pp. 652-660.

[11] Tarditi, A. G., and Shebalin, J. V., 2003 "Magnetic Nozzle Plasma Exhaust Simulation for the VASIMR Advanced Propulsion Concept," Proceedings of the 28th International Electric Propulsion Conference.

[12] Riley, B. M., Girimaji, S. S., and Richard, J. C., 2009, "Magnetic Field Effects on Axis-Switching and Instabilities in Rectangular Plasma Jets," Flow, Turbul. Combust., 82(3), pp. 375-390.

[13] Macheret, S. O., Shneider, M. N., and Miles, R. B., 2002, "Magnetohydrodynamic Control of Hypersonic Flows and Scramjets Using Electron Beam Ionization," AIAA J., 40(1), pp. 74-81.

[14] Biskamp, D., 2003, Magnetohydrodynamic Turbulence, Cambridge University Press, Cambridge, UK.

[15] Balsara, D., and Pouquet, A., 1999, "The Formation of Large-Scale Structures in Supersonic Magnetohydrodynamic Flows," Phys. Plasmas, 6, pp. 89-100.

[16] Chen, F. F., 1984, Introduction to Plasma Physics and Controlled Fusion, 2nd ed., Springer, New York.

[17] Christensson, M., Hindmarsh, M., and Brandenburg, A., 2001, "Inverse Cascade in Decaying Three-Dimensional-Magnetohydrodynamic Turbulence," Phys. Rev. E, 64(5), p. 056405.

[18] Frisch, U., Pouquet, A., Léorat, J., and Mazure, A., 1975, "Possibility of an Inverse Cascade of Magnetic Helicity in Magnetohydrodynamic Turbulence," J. Fluid Mech., 68(4), pp. 769-778.

[19] Knaepen, B., Kassinos, S. C., and Carati, D., 2004, "Magnetohydrodynamic Turbulence at Moderate Magnetic Reynolds Numbers," J. Fluid Mech., 513, pp. 199-220.

[20] Knaepen, B., and Moreau, R., 2008, "Magnetohydrodynamic Turbulence at Low Magnetic Reynolds Number," Ann. Rev. Fluid Mech., 40, pp. 25-45.

[21] Matthaeus, W. H., Ghosh, S., Oughton, S., and Roberts, D. A., 1996 "Anisotropic Three-Dimensional MHD Turbulence," J. Geophys. Res. Space Phys., 101(A4), pp. 7619-7629.

[22] Miller, R. S., Mashayek, F., Adumitoraie, V., and Givi, P., 1996, "Structure of Homogeneous Nonhelical Magnetohydrodynamic Turbulence,” Phys. Plasmas, 3(9), pp. 3304-3317.

[23] Ponty, Y., Mininni, P. D., Montgomery, D. C., Pinton, J.-F., Politano, H., and Pouquet, A., 2005. "Numerical Study of Dynamo Action at Low Magnetic Prandtl Numbers," Phys. Rev. Lett., 94(164502), p. 164502.

[24] Müller, W. C., and Grappin, R., 2005, "Spectral Energy Dynamics in Magnetohydrodynamic Turbulence," Phys. Rev. Lett., 95, p. 114502.

[25] Richard, J., Riley, B., and Girimaji, S., 2011, "Magnetohydrodynamic Turbulence Decay Under the Influence of Uniform or Random Magnetic Fields," J. Fluids Eng., 133, p. 081205

[26] Shebalin, J. V., Matthaeus, W. H., and Montgomery, D. C., 1983, “Anisotropy in MHD Turbulence Due to a Mean Magnetic Field," J. Plasma Phys., 29, pp. 525-547.

[27] Shebalin, J. V., 2005, "Theory and Simulation of Real and Ideal Magnetohydrodynamic Turbulence," Discrete Contin. Dyn. Syst., Ser. B, 5(1), p. 153174.

[28] Shebalin, J., 2009, "Plasma Relaxation and the Turbulent Dynamo," Phys. Plasmas, 16, p. 072301

[29] Yoshizawa, A., Itoh, S.-I., and Itoh, K., 2003, Plasma and Fluid Turbulence: Theory and Modeling, Institute of Physics, London.

[30] Alfvén, H., 1942, "Existence of Electromagnetic-Hydrodynamic Waves," Nature, 150(3805), pp. 405-406
[31] d'Humiéres, D., Ginzburg, I., Krafczyk, M., Lallemand, P., and Luo, L.-S., 2002, "Multiple-Relaxation-Time Lattice Boltzmann Models in Three Dimensions," Philos. Trans. R. Soc. Lond. A, 220, pp. 437-451.

[32] Eggels, J. G. M., 1996, "Direct and Large-Eddy Simulation of Turbulent Fluid Flow Using the Lattice-Boltzmann Scheme," Int. J. Heat Fluid Flow, 17, pp. 307-323.

[33] Girimaji, S. S., 2007, "Boltzmann Kinetic Equation for Filtered Fluid Turbulence," Phys. Rev. Lett., 99, p. 034501.

[34] He, X., and Luo, L.-S., 1997, "A Priori Derivation of the Lattice Boltzmann Equation,” Phys. Rev. E., 55, pp. R6333-R6337.

[35] He, X., and Luo, L.-S., 1997, "Theory of the Lattice Boltzmann Method: From the Boltzmann Equation to the Lattice Boltzmann Equation a Priori Derivation of the Lattice Boltzmann Equation," Phys. Rev. E. 56, p. 6811-6817.

[36] Lee, K., Yu, D., and Girimaji, S. S., 2006, "Lattice Boltzmann DNS of Decaying Compressible Isotropic Turbulence With Temperature Fluctuations," Int. J. Comput. Fluid Dyn., 20(6), pp. 401-413.

[37] Luo, L.-S., 1998, "Unified Theory of Lattice Boltzmann Models for Nonideal Gases," Phys. Rev. Lett., 81, pp. 1618-1621.

[38] Luo, L.-S., and Girimaji, S. S., 2003, "Theory of the Lattice Boltzmann Method: Two-Fluid Model for Binary Mixtures," Phys. Rev. E., 67, p. 036302.

[39] Shan, X., and Doolen, G. D., 1993, "Lattice Boltzmann Model for Simulating Flows With Multiple Phases and Components," Phys. Rev. E., 47(3), pp. 1815-1819.

[40] Martys, N. S., Shan, X., and Chen, H., 1998, "Evaluation of the External Force Term in the Discrete Boltzmann Equation," Phys. Rev. E., 58(5), pp. 6855-6857.

[41] Yu, H., Girimaji, S., and Luo, L., 2005, "Lattice Boltzmann Simulations of Decaying Homogeneous Isotropic Turbulence," Phys. Rev. E, 71(1), p. 016708.

[42] Yu, D., and Girimaji, S., 2005, "DNS of Homogenous Shear Turbulence Revisited with the Lattice Boltzmann Method," J. Turbul. 6(6), pp. 1-17.

[43] Yu, H., Girimaji, S. S., and Luo, L.-S., 2005, "DNS and LES of Decaying Isotropic Turbulence With and Without Frame Rotation using Lattice Boltzmann Method," J. Comput. Phys., 209(2), pp. 599-616.

[44] Yu, D., and Girimaji, S. S., 2006, "Direct Numerical Simulations of Homogeneous Turbulence Subject to Periodic Shear,” J. Fluid Mech., 566, pp. 117-151.

[45] Yu, H., and Girimaji, S. S., 2005, "Near-Field Turbulent Simulations of Rectangular Jets Using Lattice Boltzmann Method," Phys. Fluids, 17(12), p. 125106.

[46] Dellar, P., 2002, "Lattice Kinetic Schemes for MHD," J. Comput. Phys., 179, pp. $95-126$.

[47] Riley, B. M., Richard, J. C., and Girimaji, S. S., 2008, "Assessment of Magnetohydrodynamic Lattice Boltzmann Schemes in Turbulence and Rectangular Jets," Int. J. Modern Phys. C, Comput. Phys. Phys. Comput., 18(8), pp. $1211-1220$.

[48] Riley, B. M., 2007, "Magnetohydrodynamic Lattice Boltzmann Simulations of Turbulence and Rectangular Jet Flow," M.S. thesis, Texas A\&M University, College Station, TX.

[49] Krafczyk, M., Tölke, J., and Luo, L.-S., 2003, "Large-Eddy Simulations With Multiple-Relaxation-Time LBE Model," Int. J. Mod. Phys. B, 17, pp. 33-39.

[50] Chen, S., and Doolen, G., 2003, "Lattice Boltzmann Method for Fluid Flows," Ann. Rev. Fluid Mech., 30(1), pp. 329-364.

[51] Dong, Y., and Sagaut, P., 2008, "A Study of Time Correlations in Lattice Boltzmann-Based Large-Eddy Simulation of Isotropic Turbulence," Phys. Fluids, 20, p. 035105.

[52] Premnath, K., Pattison, M., and Banerjee, S., 2009, "Dynamic Subgrid Scale Modeling of Turbulent Flows Using Lattice-Boltzmann Method," Phys. A: Stat Mech. Appl., 388(13), pp. 2640-2658.

[53] Kerimo, J., and Girimaji, S., 2007, "Boltzmann-BGK Approach to Simulating Weakly Compressible 3D Turbulence: Comparison Between Lattice Boltzmann and Gas Kinetic Methods," J. Turbul., 8(1), pp. 1-16.

[54] Gekelman, W., Vincena, S., and Collette, A., 2008, "Visualizing ThreeDimensional Reconnection in a Colliding Laser Plasma Experiment," IEEE Trans. Plasma Sci., 36(4), pp. 1122-1123.

[55] Mininni, P., Lee, E., Norton, A., and Clyne, J., 2008, "Flow Visualization and Field Line Advection in Computational Fluid Dynamics: Application to Magnetic Fields and Turbulent Flows," New J. Phys., 10, p. 125007.

[56] Zhang, Y., Boehmer, H., Heidbrink, W., McWilliams, R., Leneman, D., and Vincena, S., 2007, "Lithium Ion Sources for Investigations of Fast Ion Transport in Magnetized Plasmas,” Rev. Sci. Instrum., 78, p. 013302.

[57] Drozdenko, T., and Morales, G., 2001, "Nonlinear Effects Resulting From the Interaction of a Large-Scale Alfvén Wave With a Density Filament," Phys. Plasmas, 8, pp. 3265-3277. 